ARTICLE

\title{
Establishment of human iPSC-based models for the study and targeting of glioma initiating cells
}

Ignacio Sancho-Martinez ${ }^{1, *, \dagger}$, Emmanuel Nivet ${ }^{1, *, \dagger}$, Yun Xia ${ }^{1, *, \dagger}$, Tomoaki Hishida ${ }^{1}$, Aitor Aguirre ${ }^{1}$ Alejandro Ocampo ${ }^{1}$, Li Ma1,2, Robert Morey³ ${ }^{3}$ Marie N. Krause ${ }^{1}$, Andreas Zembrzycki ${ }^{4}$, Olaf Ansorge ${ }^{5}$, Eric Vazquez-Ferrer ${ }^{1}$, Ilir Dubova1,2, Pradeep Reddy ${ }^{1}$, David Lam¹, Yuriko Hishida1, Min-Zu Wu1, Concepcion Rodriguez Esteban ${ }^{1}$, Dennis O'Leary ${ }^{4}$, Geoffrey M. Wahl ${ }^{6}$, Inder M. Verma ${ }^{7}$, Louise C. Laurent ${ }^{3}$ \& Juan Carlos Izpisua Belmonte ${ }^{1}$

Glioma tumour-initiating cells (GTICs) can originate upon the transformation of neural progenitor cells (NPCs). Studies on GTICs have focused on primary tumours from which GTICs could be isolated and the use of human embryonic material. Recently, the somatic genomic landscape of human gliomas has been reported. RTK (receptor tyrosine kinase) and p53 signalling were found dysregulated in $\sim 90 \%$ and $86 \%$ of all primary tumours analysed, respectively. Here we report on the use of human-induced pluripotent stem cells (hiPSCs) for modelling gliomagenesis. Dysregulation of RTK and p53 signalling in hiPSC-derived NPCs (iNPCs) recapitulates GTIC properties in vitro. In vivo transplantation of transformed iNPCs leads to highly aggressive tumours containing undifferentiated stem cells and their differentiated derivatives. Metabolic modulation compromises GTIC viability. Last, screening of 101 anti-cancer compounds identifies three molecules specifically targeting transformed iNPCs and primary GTICs. Together, our results highlight the potential of hiPSCs for studying human tumourigenesis.

\footnotetext{
${ }^{1}$ Gene Expression Laboratory Belmonte, Salk Institute for Biological Studies, 10010 North Torrey Pines Road, La Jolla, California 92037, USA. ${ }^{2}$ Universidad Católica San Antonio de Murcia (UCAM) Campus de los Jerónimos, № 135 Guadalupe, Murcia 30107, Spain. ${ }^{3}$ Department of Reproductive Medicine, University of California, San Diego, Sanford Consortium for Regenerative Medicine, 2880 Torrey Pines Scenic Drive, La Jolla, California 92037, USA. ${ }^{4}$ Molecular Neurobiology Laboratory, Salk Institute for Biological Studies, 10010 North Torrey Pines Road, La Jolla, California 92037, USA. ${ }^{5}$ Department of Neuropathology, West Wing, Level 1, John Radcliffe Hospital, Oxford OX3 9DU, UK. ${ }^{6}$ Gene Expression Laboratory Wahl, Salk Institute for Biological Studies, 10010 North Torrey Pines Road, La Jolla, California 92037, USA. ${ }^{7}$ Laboratory of Genetics, Salk Institute for Biological Studies, 10010 North Torrey Pines Road, La Jolla, California 92037, USA. * These authors contributed equally to this work. †Present addresses: Centre for Stem Cells and Regenerative Medicine, King's College London, 28th Floor, Tower Wing, Guy's Hospital, Great Maze Pond, London SE1 9RT, UK (I.S.-M.); Aix Marseille Université, CNRS, NICN UMR 7259, Marseille 13344, France (E.N.); Lee Kong Chian School of Medicine, Experimental Medicine Building, 59 Nanyang Drive, Singapore 636921, Singapore (Y.X.). Correspondence and requests for materials should be addressed to J.C.I.B. (email: belmonte@salk.edu).
} 
A dult gliomas are the most malignant human brain tumours $^{1}$, with no curative therapy available. Gliomas can originate as a result of adult NPCs transformation to glioma tumour-initiating cells (GTICs) ${ }^{1,2}$. However, strategies for studying the mechanisms underlying the transformation of adult human NPCs to GTICs remain scarce with most mechanistic studies relying on the use of transgenic murine models ${ }^{1}$. Recent reports have highlighted the potential of reprogramming to induce the conversion of differentiated glioma cells to a GTIC-like phenotype ${ }^{3}$. Despite much success, reprogramming of cancer cells to GTICs requires the use of already transformed cells isolated from a pre-existent tumour ${ }^{1,3-10}$. Thus, and similar to the use of primary glioma cells, such reprogrammed GTICs prevent functional studies on the mechanisms leading to NPC transformation and tumour initiation. Accordingly, functional studies on NPC transformation and GTIC formation remain largely limited to the use of differentiated neural derivatives ${ }^{11,12}$ and/or the use of murine models ${ }^{1,13,14}$. Contrary to murine models, isolation of adult human NPCs remains restricted to brain tissue material obtained from patients with pathological conditions, such as epilepsy, or post-mortem. As an alternative to study human gliomagenesis, the use of primary fetal NPCs and NPCs differentiated from human embryonic stem cells has been reported ${ }^{1,2,10,15,16}$. However, the use of embryonic/fetal material remains the subject of ethical controversy and limits the possibility for investigating the role of different mutations in various genetic backgrounds represented in the human population.

In 2006, Kazutoshi Takahashi and Shinya Yamanaka were able to reprogram somatic cells into pluripotent stem cells upon the forced expression of a small number of defined genes ${ }^{17}$. Reprogramming to human-induced pluripotent stem cells (hiPSCs) possesses the inherent advantages of voiding the need for embryonic material while allowing for the generation of pluripotent cells from any given genetic background in a patient-specific manner. The possibility for generating patientspecific iPSCs holds great promise for the future development of autologous cell therapies as well as open unprecedented opportunities for disease modelling and drug discovery studies $^{18}$. In addition, modelling of complex phenotypes, such as aging, can be accomplished by overexpressing specific mutant genes in otherwise wild-type hiPSCs ${ }^{19,20}$. Therefore, the use of hiPSCs, and/or their derivatives, in which defined genetic alterations related to cancer are introduced might represent a suitable strategy for the establishment of human cancer models.

Here we report on the establishment of tractable in vitro and in vivo hiPSC models for the study of human iNPC transformation to GTIC-like cells. Genetic manipulation of p53 and receptor tyrosine kinase signalling leads to the acquisition of cancer stem cell-like features in vitro, including enhanced self-renewal and migratory properties alongside metabolic reprogramming. Transformation of iNPCs to GTIC-like cells leads to the generation of human glioma-like tumours with distinct histopathological features upon orthotopic transplantation of 500 cells into the murine brain as well as in serial transplantation experiments. Ultimately, our results highlight the suitability of human iPSC technologies for the modelling of human cancer.

\section{Results}

Transformation of human iPSC-derived neural progenitors. Mutations affecting components of the p53 signalling pathway are collectively observed in $\sim 86 \%$ of all human gliomas ${ }^{21}$ Therefore, we first generated hiPSC lines from healthy/'wild-type' human fibroblasts (hereafter referred to as ${ }_{\text {wT }}$ iPSCs) and lines in where p53 was knocked down (hereafter referred to as p53KDiPSCs) (Supplementary Fig. 1a). All hiPSCs presented the typical hallmarks of pluripotency including in vivo teratoma formation in the absence of apparent malignant transformation (Supplementary Fig. 1b-f). Next, we differentiated NPCs from the generated hiPSCs (Supplementary Fig. 2a). Immunofluorescence analysis as well as multilineage differentiation potential confirmed the NPC identity of the differentiated cells (hereafter referred to as iNPCs) (Supplementary Fig. 2b-d). We have previously reported that human glioma infiltration is driven by activation of Src-family kinases (SFKs) and targeting SFKs has emerged as an attractive therapeutic approach currently under development ${ }^{22-25}$. In addition, Brennan et al. ${ }^{21}$ described mutations collectively leading to PI3K and MAPK hyperactivation in $\sim 90 \%$ of human gliomas. Therefore, we additionally generated iNPCs-overexpressing mutant-active versions of Src (to mimic infiltrative behaviour), EGFR (duplicated and/or mutated in $\sim 57 \%$ of human gliomas) ${ }^{21}$ and Ras (mutated in $\sim 1 \%$ of human gliomas and a common upstream regulator of both PI3K and MAPK signalling) ${ }^{21}$. Lentivirus-mediated overexpression of the different mutant

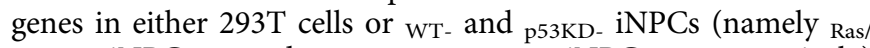
EGFR/SrciNPCs and p53KD-Ras/EGFR/SrciNPCs, respectively) confirmed the hyperactivation of PI3K and MAPK pathways as demonstrated by increased AKT and ERK phosphorylation, respectively (Supplementary Fig. 2a,e,f).

GTICs were first isolated based on the expression of CD133 and thus CD133 + thought to unequivocally label glioma cells with stem cell properties ${ }^{26}$. However, CD133 - glioma cells presenting stem cell and GTIC properties have also been reported $^{2 \%, 28}$ and CD133 + cells found in a variety of normal tissues other than tumours ${ }^{29}$. Nevertheless, we sought to investigate the role of putative GTIC surface markers for enriching cancer populations with stem cells properties. On the basis of our flow cytometry results (Fig. 1a), we first separated $\mathrm{CD} 133$ + and CD133 - cells and performed in vitro single-cell tumour forming assays. To avoid limiting our analyses to a single marker, we also sorted out CD15 + and CD15 - cells as well as CXCR4 + and CXCR4 - populations. All different cell populations demonstrated comparable colony forming potential (Supplementary Fig. 2g). These results are in agreement with the notion that GTICs are very heterogeneous. Indeed, a universally accepted panel of markers for the characterization and isolation

\footnotetext{
Figure 1 | Transformation of human iNPCs results in the acquisition of a GTIC-like phenotype in vitro. (a) Flow cytometry analysis for the indicated markers in different transformed and wild-type iNPC groups ( $n=4$ /group with technical duplicates). (b) Transformation of human iNPCs leads to increased self-renewal properties only in cells where PI3K and MAPK signalling is dysregulated by overexpression of Ras/EGFR/Src mutant genes as highlighted by single-cell self-renewal assays ( $n=3$ /group with 24 technical replicates). (c) iNPC transformation results in the upregulation of endogenous NANOG as highlighted by western blot analyses. (d) NANOG expression is upregulated in primary human adult glioma samples. (e) Unsupervised cluster analysis of mRNA expression data highlighting the similarities between transformed iNPCs and primary GTICs. Please note that ${ }^{553 K D i N P C s}$ are more similar to wTiNPCs than to primary GTICs. ( $\mathbf{f}, \mathbf{g})$ Transformation of human iNPCs induces glycolytic (f) and oxidative phosphorylation changes as indicated by Seahorse analysis $(\mathbf{g})$, as measured by ECAR and OCR $(n=4$ /group with four technical replicates). Data are represented as mean $\pm \mathrm{s}$.d. $P$ values were calculated by Student's $t$-test or Mann-Whitney test when appropriate and represented as follows: ${ }^{\star} P<0.05$. Scale bars, $200 \mu \mathrm{m}$ (d).
} 
a

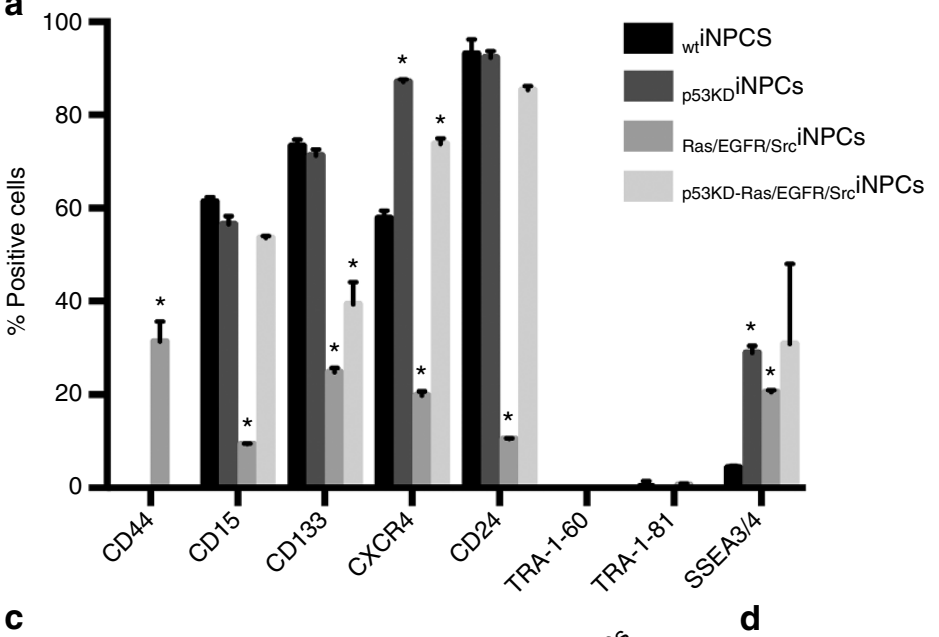

c

d b

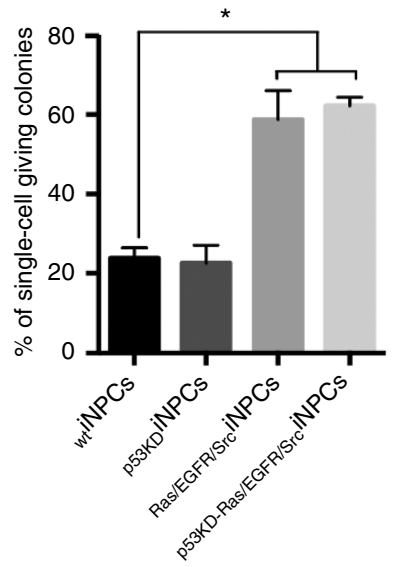

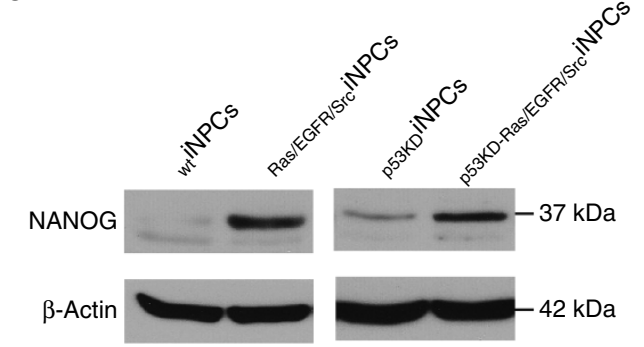

e

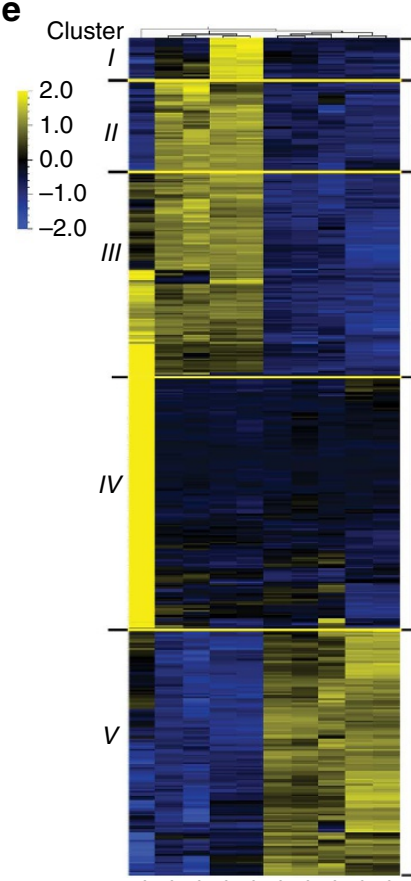

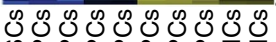

w之z之之之n

竞咅
Cancer ( $P$ value 1.27 E-03)

Cellular movement ( $P$ value $2.14 \mathrm{E}-05)$

Nervous system development

and function ( $P$ value $2.97 \mathrm{E}-04)$

Cancer ( $P$ value $3.52 \mathrm{E}-09)$

Transcriptional regulatory network in

embryonic stem cells ( $P$ value $1.43 \mathrm{E}-04)$

Cancer ( $P$ value $1.37 \mathrm{E}-08)$

Cellular growth and proliferation

( $P$ value $2.07 \mathrm{E}-08)$
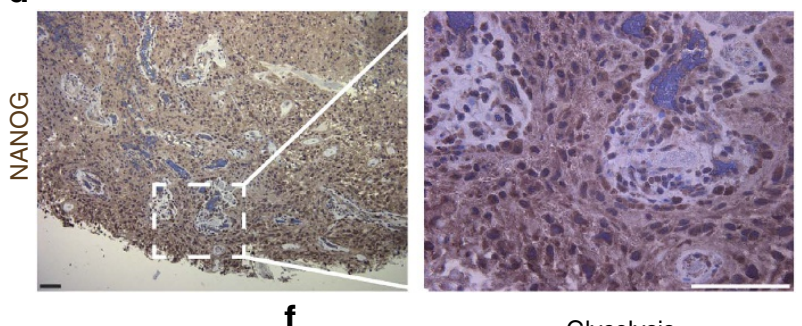

f

* Glycolysis

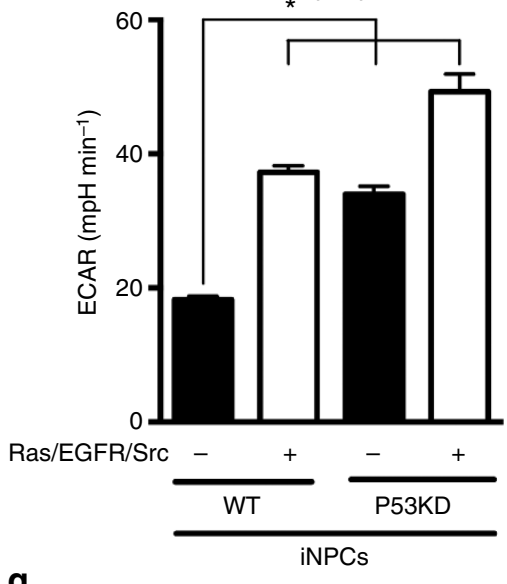

g Oxidative phosphorylation

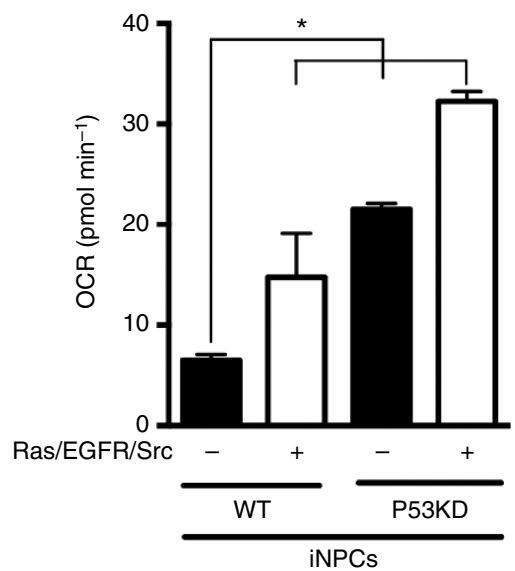


of GTICs is yet to be reported ${ }^{30}$. Variability in surface marker expression in cancer cells bearing stem cell properties is not exclusive to gliomas and has now been observed in a variety of human tumours $1,3-7,9,10$. Because of surface marker heterogeneity and their expression in certain non-transformed adult stem cells, recent reports advocate for the characterization of cancer stem cells based on functional properties, such as multilineage differentiation potential and tumour formation upon serial transplantation ${ }^{1,3-7,9,10}$. Accordingly, we next decided to investigate the self-renewal potential of non-sorted iNPCs by performing single-cell assays. Enhanced self-renewal properties were observed in all iNPCs where PI3K/MAPK signalling was dysregulated (Fig. 1b). Furthermore, NANOG, a protein expressed in iPSCs, was found upregulated in transformed iNPCs (Fig. 1c). Interestingly, immunohistochemical analyses of human brain tumour samples further confirmed NANOG expression in grade IV gliomas (Fig. 1d). Lack of TRA1-60 and TRA1-81 expression (Fig. 1a) further demonstrated that NANOG expression was due to transformation and not the presence of undifferentiated hiPSCs.

Transformation of human NPCs generates GTIC-like cells. We next compared the generated iNPCs with primary GTICs, isolated and characterized by Pollard et al. ${ }^{5}$, by performing genome-wide analyses. Genome-wide expression data demonstrated that transformed iNPCs closely resemble primary GTICs at the molecular level, though some differences were readily observed among the different groups. wTiNPCs were most similar to ${ }_{\mathrm{p} 53 \mathrm{KD}} \mathrm{iNPCs}$, while Ras/EGFR/SrciNPCs and $\mathrm{p} 53 \mathrm{KD}$-Ras/EGFR/ SrciNPCs were very similar to each other and intermediate between the ${ }_{\mathrm{wT}} \mathrm{iNPC} /{ }_{\mathrm{p} 53 \mathrm{KD}} \mathrm{iNPC}$ cluster and primary GTICs (Supplementary Data 1, analysis of the variance (ANOVA) $q$ value $<0.01$ and variance filter $=0.2$ using Qlucore). The corresponding heatmap showed five large clusters of genes that were differentially expressed among wTiNPCs, p53KDiNPCs, Ras/EGFR/SrciNPCs, p53KD-Ras/EGFR/SrciNPCs and GTICs (Fig. 1e, clusters I-V and Supplementary Data 2). Importantly, genes in cluster $\mathrm{V}$ showed higher expression in the GTICs, Ras/EGFR/SrciNPCs and p53KD-Ras/EGFR/Sri iNPCs compared with ESCs, wTiNPCs, and p53KDiNPCs (Fig. 1e, cluster V and Supplementary Data 2). Of the five clusters, cluster V showed the highest number of significant enrichments on pathways analysis, and was enriched for several cell growth-, proliferationand cancer-associated pathways. Notably, DNA methylation analysis confirmed a more undifferentiated phenotype for Ras/EGFR/SrciNPCs and p53KD-Ras/EGFR/SrciNPCs (Supplementary Table 1).

Changes in the energetic metabolism are generally observed in cancer cells, the so-called Warburg effect ${ }^{31}$. In agreement, Seahorse analyses demonstrated the clear metabolic reprogramming of transformed iNPCs (Fig. 1f,g). RNA expression analyses highlighted the upregulation of glycolytic genes typically associated with metabolic alterations in GTICs in the transformed iNPCs, including: PKM2; HK; GLUT3 (a gene recently described as critical for glucose uptake in GTICs) $^{6}$; and $L D H B$ (a gene commonly upregulated in gliomas bearing IDH mutations) ${ }^{32}$ (Supplementary Fig. 3a). Higher lactate production and lower glucose consumption in transformed iNPCs revealed significant differences in the lactate/glucose ratio (Supplementary Fig. 3b). Non-labelled mass spectrometry highlighted, as before, higher similarities between $\mathrm{p}_{53 \mathrm{KD}^{-}}$and ${ }_{\mathrm{WT}} \mathrm{iNPCs}$, an intermediate profile in $\mathrm{Ras}_{\mathrm{EGFR} / \mathrm{Src}} \mathrm{iNPCs}$ and marked differences in the levels of metabolites involved in carbon and aminoacid metabolism in p53KD-Ras/EGFR/SriNPCs (Supplementary Fig. 3c). Next, U-13C glucose labelling was used to monitor carbon flux in the transformed iNPCs as compared with wTiNPCs. No significant differences in levels of production of glycolytic metabolites were found among the four lines, except for increased glucose flux into lactate and glycine in p53KD-Ras/EGFR/SrciNPCs, (Supplementary Fig. 3d). Interestingly, we observed much lower influx of glucose into glutamate production and into the trichloroacetic acid or tricarboxylic acid cycle (as highlighted by differential labelling of aspartate, malate, fumarate and $\alpha$-ketoglutarate) in Ras/EGFR/SrciNPCs and p53KD-Ras/EGFR/SrciNPCs, compared with $\mathrm{wT}^{-}$and ${ }^{\mathrm{i}} 53 \mathrm{KD} \mathrm{iNPCs}$, which shared a similar metabolic profile (Supplementary Fig. 3d). Quantification of ROS levels further highlighted metabolic reprogramming and demonstrated reduced mitochondrial ROS formation upon iNPC transformation, whereas total ROS levels remained comparable across all different groups (Supplementary Fig. 4a,b). MitoTracker analysis indicated that disruption of signalling pathways resulted in morphological mitochondrial changes and higher fragmentation of mitochondrial networks as compared with wTiNPCs (Supplementary Fig. 4c).

To test whether transformation of iNPCs resulted in the functional acquisition of GTIC-like properties in vivo ${ }^{4,7,9}$, we performed xenograft transplantation studies. Orthotopic injection of transformed iNPCs (500,000 cells) into the murine brain resulted in the rapid appearance of highly aggressive tumours (Fig. 2a and Supplementary Table 2). Tumours were then analysed for the presence of classical hallmarks of human glioblastoma multiforme (GBM) such as pleomorphic GFAP + glial cells, mitoses, diffuse infiltrative growth, pseudopalisading microscopic necrosis and microvascular proliferation (Fig. 2b-d and Supplementary Fig. 5a,b). Immunofluorescence analysis demonstrated the presence of de-differentiated NESTIN + and SOX $2+$ cancer cell populations as well as oligodendrocytes $(\mathrm{O} 4)$, astrocytes (GFAP) and neurons (TUJ-1) confirming the in vivo differentiation potential of the generated GTIC-like cells (Fig. 2c and Supplementary Fig. 5b). In addition to the presence of undifferentiated tumour cells, all tumours presented mitotic figures. Thus, these data suggested a pathological classification as high-grade astrocytomas (grades III-IV). In line with the differences observed in vitro, in vivo analyses highlighted intergroup differences reflecting different glioma subtypes.

\footnotetext{
Figure 2 | Transformation of human iNPCs results in the acquisition of GTIC-like properties in vivo. (a) Representative pictures demonstrating the formation of vascularized tumours only in animals receiving transformed, and not wild-type, human iNPCs. (b) Haematoxylin-eosin staining demonstrating the presence of highly aggressive brain tumours upon orthotopic transplantation of transformed human iNPCs into the murine brain. (c) Immunofluorescence analysis demonstrating the presence of undifferentiated SOX2 + cells (red) as well as differentiation into the three major neural lineages upon transplantation of transformed human iNPCs. Please note the higher cellularity in tumours derived upon transplantation of p53KD-Ras/EGFR/SrciNPCs. HUNU (green) indicates human nuclear antigen staining; O4 (green) indicates oligodendrocyte differentiation; Tuj1 (red) indicates neuronal differentiation; and GFAP (red) indicates glial differentiation. pERK (purple) and pAKT (purple) were only detected in tumours generated upon transplantation of iNPCs-overexpressing mutant versions of Ras/EGFR/Src. Cells were counterstained with DAPI (blue) and their proliferative state monitored by Ki67

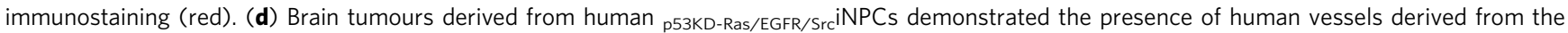
injected GTIC-like cells as indicated by CD31 and HUNU co-localization. $n=5$ animals/condition. A minimum of three brain sections/animal/condition were analysed. Scale bars, $200 \mu \mathrm{m}$ (b); $100 \mu \mathrm{m}$ or $25 \mu \mathrm{m}$ as indicated (c); and $25 \mu \mathrm{m}$ (d).
} 
Tumours arising from ${ }^{\mathrm{p} 53 \mathrm{KD}} \mathrm{iNPCs}$ were reminiscent of 'small-cell' GBM, showing elongated spindle cells with eosinophilic cytoplasm alternating with clusters of compact cells with little cytoplasm and a high nuclear:cytoplasmic ratio. These tumours displayed a diffuse growth pattern but lacked the pleomorphism usually seen in GBM and no obvious transition zone to normal brain was detected. Brain tumours developed upon injection of Ras/EGFR/SrciNPCs showed a nodular growth a ${ }_{w i}$ iNPCs

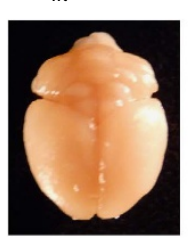

C
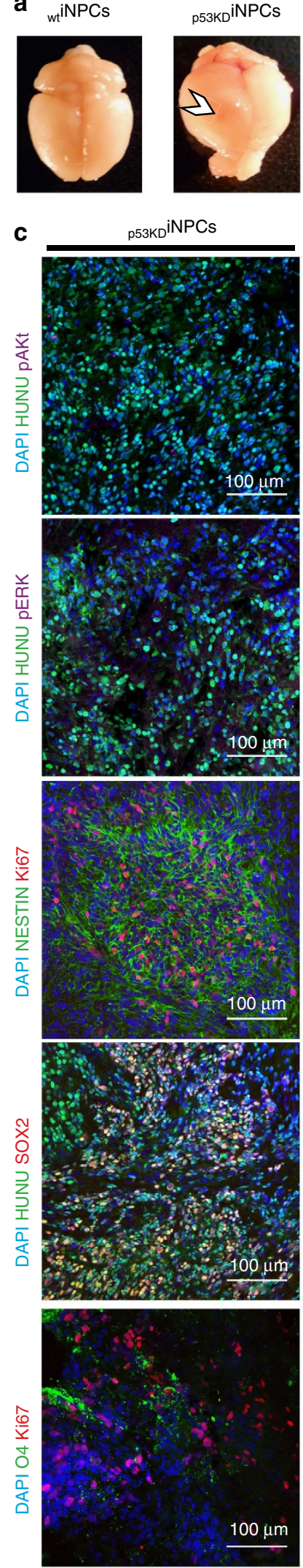

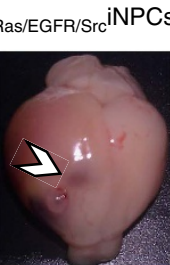

p53KD-Ras/EGFR/Src iNPCs
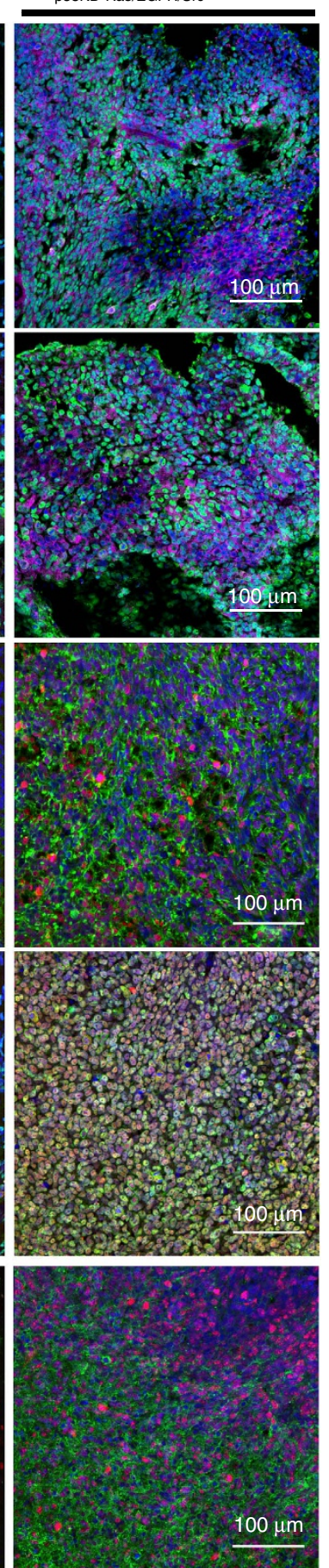

b

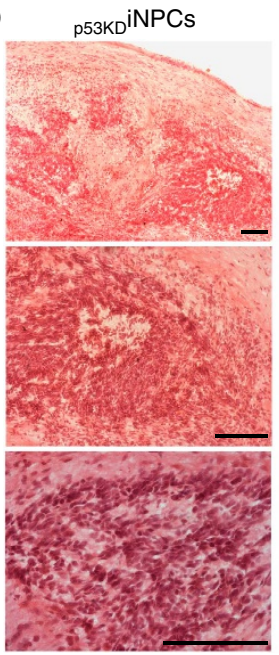

${ }_{\mathrm{p} 53 \mathrm{KD}}^{\mathrm{iNPCs}}$
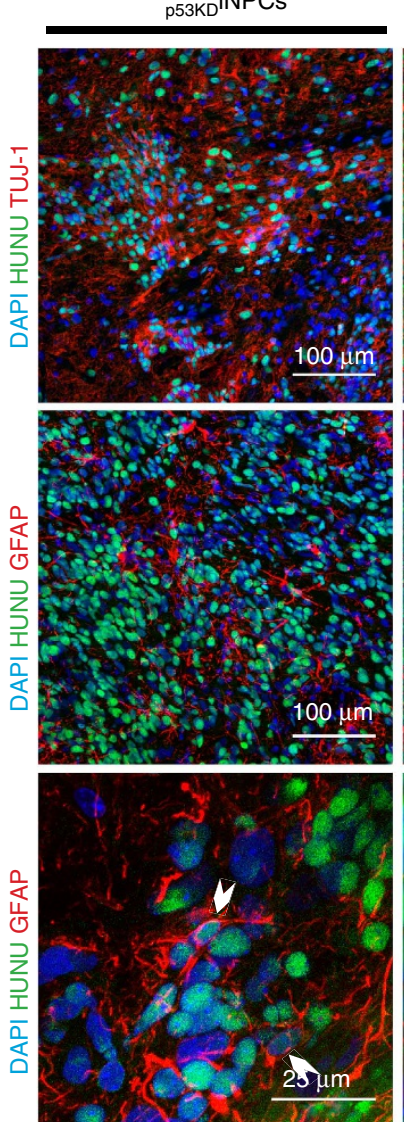

d

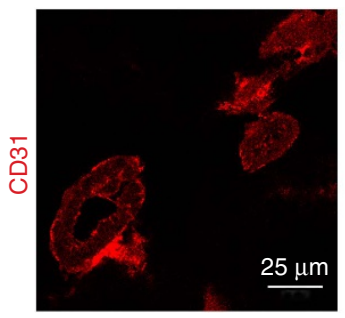

p53KD-Ras/EGFR/Src iNPCs

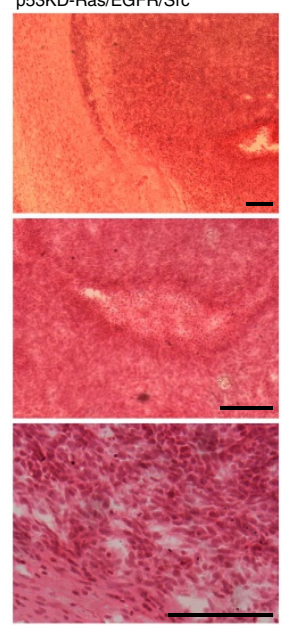

p53KD-Ras/EGFR/SrciNPCs
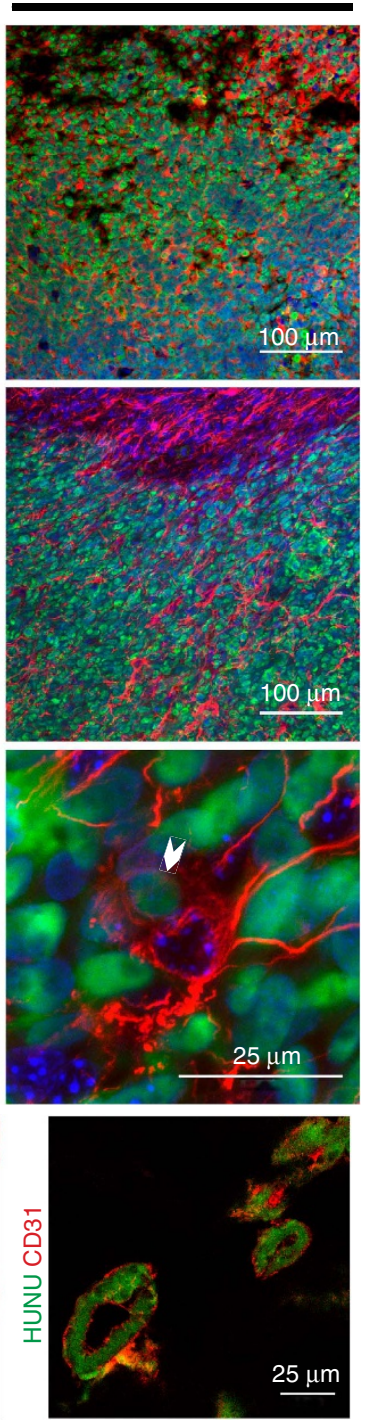
with perivascular spread but not much diffuse parenchymal invasion was observed. Interestingly, some necrosis was observed but no pseudopalisading. Notably, the pale cytoplasm and elongated shape that were observed are reminiscent of mesenchymal morphology. Lastly, from all three groups analysed, the tumours developed after injection of ${ }_{\text {p53KD-Ras/EGFR/SrciNPCs }}$ closely resembled grade IV glioblastomas. These tumours demonstrated the highest cellularity and massive necrotic areas as well as microvascularization, pseudopalisading, breaching of the pial surface and invasion of the subarachnoid space as seen in GBM and gliosarcomas. Most notably, transdifferentiated human $\mathrm{CD} 31+$ endothelial cells a feature of human gliomas for promoting tumour vascularization ${ }^{33}$, were only observed upon injection of p53KD-Ras/EGFR/SrciNPCs (Fig. 2d). Animals injected with ${ }_{\text {WT }} \mathrm{iNPC}$ lived their normal lifespan and did not present brain tumours (Supplementary Table 2) whereas injection of undifferentiated iPSCs led to the formation of well-defined embryonic-like teratomas histologically distinct from the brain tumours generated upon transplantation of transformed iNPCs (Supplementary Fig. 1e). Next, we performed xenograft transplantation experiments with limited cell numbers as well as secondary tumour formation assays. Among the transformed groups, only iNPCs dysregulated for PI3K and MAPK signalling (Ras/EGFR/SrciNPCs and p53KD $^{-}$Ras/EGFR/SrciNPCs) were able to form primary tumours when 500 cells were orthotopically injected into the murine brain (Supplementary Fig. 6a and Supplementary Table 3). Furthermore, serial transplantation of Ras/EGFR/SrciNPCs and $\mathrm{p}_{3} \mathrm{KD}^{-} \mathrm{Ras} / \mathrm{EGFR} / \mathrm{Src} \mathrm{iNPCs}$ resulted in the formation of highly aggressive and infiltrative secondary tumours, presenting similar histological characteristics to those observed in the respective primary tumours, even upon secondary transplantation of 500 cells (Supplementary Fig. 6b-e and Supplementary Table 3). In agreement with the similarities between ${ }_{\mathrm{p} 53 \mathrm{KD}} \mathrm{iNPCs}$ and wTiNPCs, p53KDiNPCs did not lead to secondary tumour formation upon serial transplantation regardless of the number of cells injected (Supplementary Table 3 ).

Transformation of iNPCs results in differential SOX2 binding. To highlight potential differences between normal iNPCs and GTICs, we analysed the binding of SOX2, a transcription factor common to both wTiNPCs and GTICs. ChIP-seq experiments demonstrated differential binding of SOX2 in primary GTICs and transformed GTIC-like cells as compared with ${ }_{\text {wT }}$ iNPCs (Fig. 3a). Correlation analysis between ChIP and mRNA expression data highlighted a total of approximately eight genes commonly regulated by SOX2 that were both differentially bound as well as differentially expressed between primary GTICs/p53KD-Ras/EGFR/ SrciNPCs as compared with wTiNPCs (Supplementary Data 3, highlighted in red). In agreement with a more undifferentiated phenotype, SOX2 preferentially bound to gene promoters associated to cell dedifferentiation and neural development processes in p53KD-Ras/EGFR/SrciNPCs. Interestingly, one gene, COX6A1, a gene blocking Bax-induced apoptosis, showed decreased SOX2 binding associated with increased expression in the GTICs and p53KD-Ras/ EGFR/SrciNPCs compared with the wTiNPCs and p53KDiNPCs (Fig. 3a). Genes for which decreased SOX2 binding was associated with decreased expression in GTICs and $533 \mathrm{KD}$-Ras/EGFR/SrciNPCs compared with wTiNPCs and ${ }_{\mathrm{p} 53 \mathrm{KD}} \mathrm{iNPCs}$ were MAP2K5/ERK5, whose activities have been associated with tumour development and the acquisition of mesenchymal stem-like properties in cancer cells $^{34}$, and C10orf67, a susceptibility locus associated with sarcoidosis and Crohn's disease (Fig. 3a).

Chemical screening highlights compounds targeting GTICs. We next tested the suitability of the generated platforms for the testing of chemicals with potential anti-cancer activities. First we investigated the GTIC dependence for hyperactive PI3K/MAPK signalling and their causative role in the acquisition of stem cell properties and metabolic reprogramming. Notably, only MAPK inhibition with PD098059 restored normal glycolytic activities, whereas inhibition of both, PI3K (LY294002) and MAPK signalling, reduced oxidative phosphorylation to the levels observed in wTiNPCs (Fig. 3b,c). In addition, chemical inhibition of PI3K and MAPK compromised stem cell self-renewal properties (Supplementary Fig. 7). Next, we focused on metabolic modulation by using: (i) Carbonyl cyanide 4-(trifluoromethoxy)phenylhydrazone (FCCP), a compound dissipating mitochondrial membrane potential; (ii) rotenone in conjunction with antimycin A (Rot/AA), both compounds targeting complexes I and III and leading to the massive production of ROS; and (iii) 2-DG, an analogue glucose acting as a suicide inhibitor. Among all metabolic modulators, inhibition of glycolysis by 2-DG, a compound previously reported for the targeting of gliomas and currently in clinical testing, significantly compromised the self-renewal properties of transformed iNPCs (Fig. 3d). Then, we performed MTS assays to assess cell viability in the presence of metabolic chemical modulators. Interestingly, FCCP did not show any consistent effect across the different groups whereas the use of Rot/AA affected cell viability in all iNPCs (Fig. 3e and Supplementary Fig. 8a). Importantly, inhibition of glycolysis demonstrated a dramatic effect across the different groups of transformed iNPCs, whereas ${ }_{\text {WT }}$ iNPCs remained largely unaffected (Fig. 3e). In view of these results, we next decided to perform a proof-of-concept screening with 101 FDA-approved anti-cancer compounds (Supplementary Fig. $8 \mathrm{~b}$ and Supplementary Table 4). MTS assays highlighted 16 different compounds compromising cell viability (Supplementary Fig. 8c and Supplementary Table 4, highlighted in red). Among the different compounds, nelarabine, letrozole and capecitabine, currently under testing for the treatment of gliomas, demonstrated specificity against transformed iNPCs and primary GTICs, whereas cabazitaxel also hampered ${ }_{\text {WT }}$ iNPC viability (Fig. 4a).

Glioma cell infiltration remains a major cause of tumour relapse and we have previously demonstrated that SFK signalling mediates basal glioma invasion by activating MMP9 (ref. 25). Two-chamber migration assays demonstrated enhanced migratory properties in transformed iNPCs (Supplementary Fig. 9a,b). RNA profiling demonstrated the significant upregulation of MMP9 in Ras/EGFR/SrciNPCs and p53KD-Ras/EGFR/Sri iNPCs (Supplementary Fig. 9c) and MMP9 inhibition significantly hampered migration (Supplementary Fig. 9b). In addition, all identified anti-cancer compounds and two metabolic inhibitors (2-DG and Rot/AA) hampered the migration of primary GTICs and transformed iNPCs (Fig. 4b). Differentiation of cancer stem cells is thought to give rise to less malignant populations that are presumably more sensitive to chemotherapy, whereas the self-renewing stem cell compartment is thought to contribute to resistance and recurrence $e^{2,3,8,9,35-37}$. Self-renewal assays indicated that Ras/EGFR/SrciNPCs and $\mathrm{p} 53 \mathrm{KD}$-Ras/EGFR/SrciNPCs possessed the highest self-renewal potential, whereas $\mathrm{p} 53 \mathrm{KD}$ iNPCs were more similar to ${ }_{\text {wT }}$ iNPCs (Supplementary Fig. 9d,e). Treatment with the identified compounds significantly compromised the self-renewal properties of Ras/EGFR/SrciNPCs and p53KD-Ras/EGFR/ SrciNPCs (Fig. 4c). In addition, iNPC-differentiated neural cells demonstrated higher susceptibility to chemical treatments (Supplementary Fig. 10a). Next, we investigated the effect of the identified compounds in established glioma lines. Treatment of U-87 MG and LN229 glioma cells with the different compounds demonstrated the efficient induction of cell death with nelarabine and letrozole, whereas capecitabine was largely ineffective (Supplementary Fig. 10b). Last, we decided to test the effect of 
a
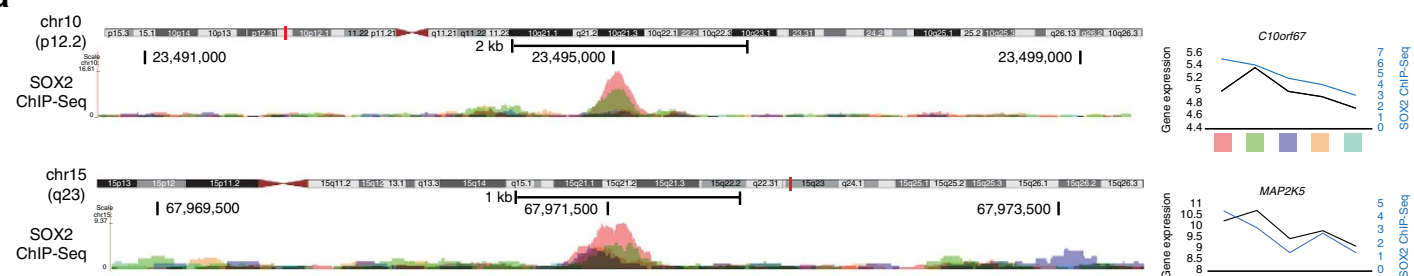

sox2

chr
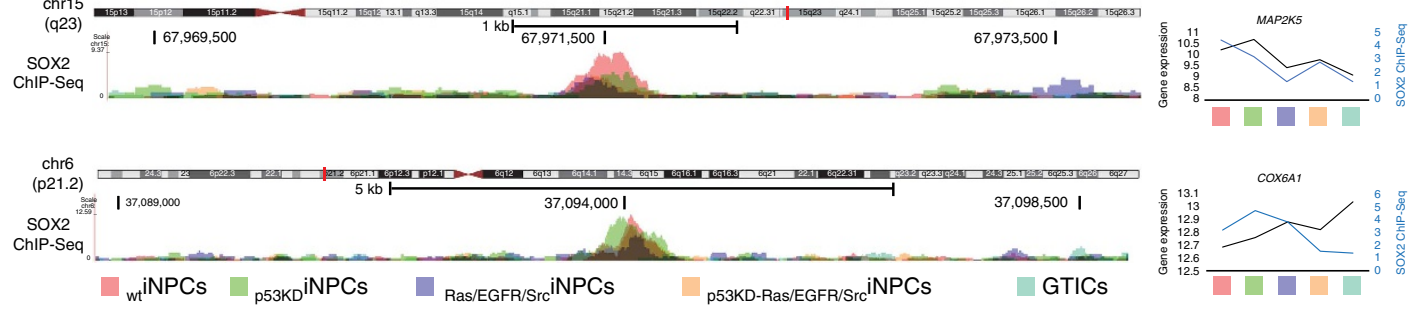

b

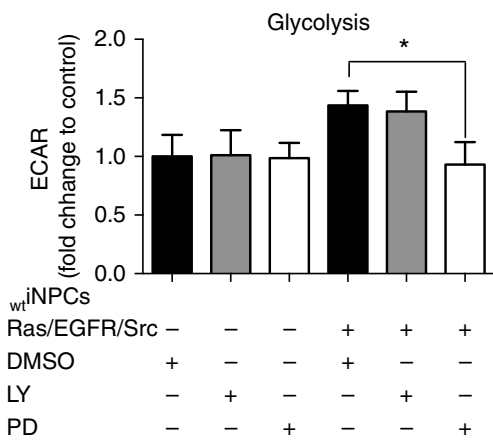

C
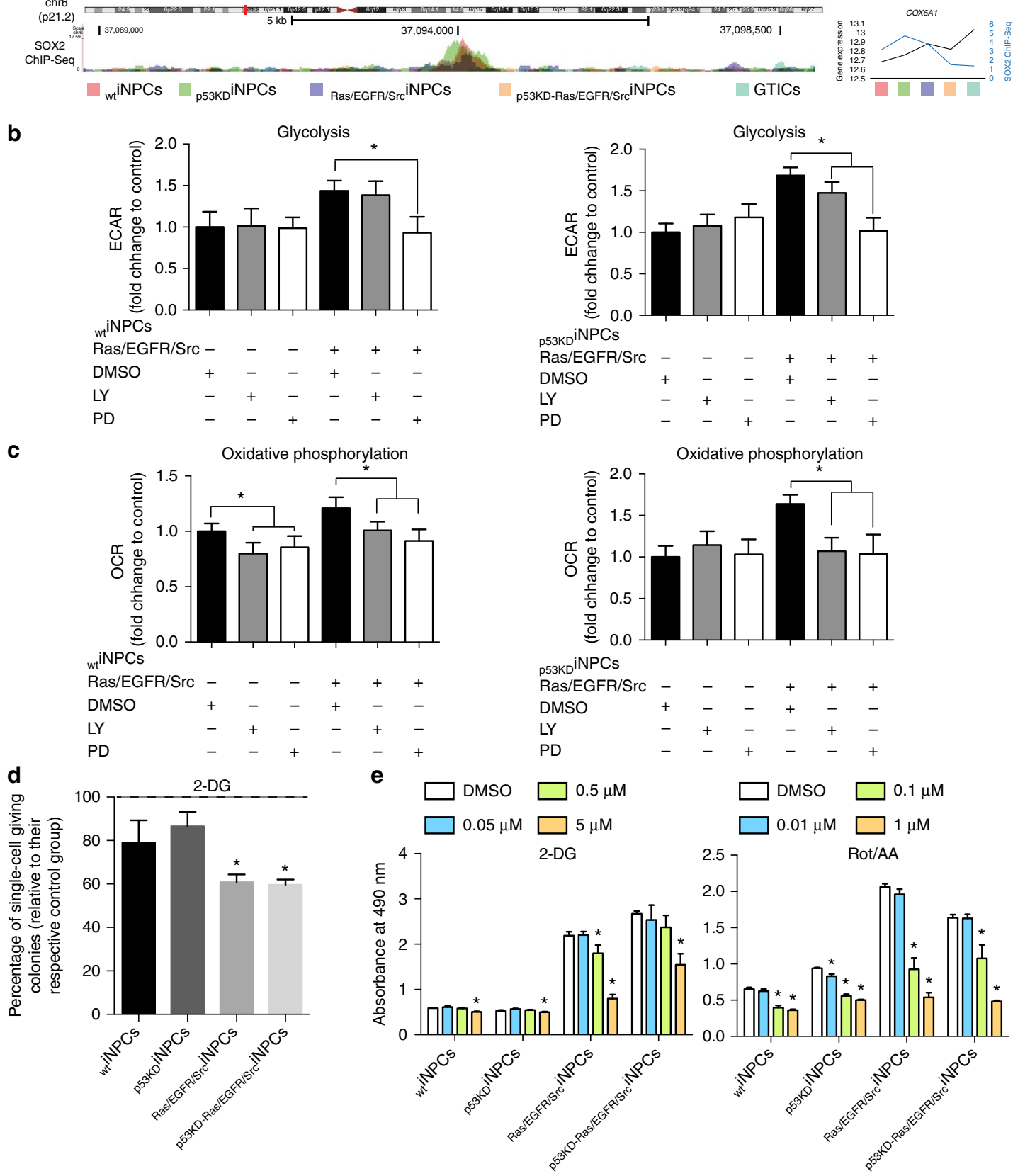

Figure 3 | Human iNPCs transformation leads to differential SOX2 binding and metabolic reprogramming. (a) Chromatin Immunoprecipitation with SOX2 antibodies followed by deep-sequencing demonstrates differential binding of SOX2 in wTiNPCs as compared with transformed iNPCs and primary GTICs. (b,c) Modulation of PI3K and MAPK signalling pathways with LY294002 (LY) and PD098059 (PD), respectively, reverts the metabolic changes observed in transformed iNPCs as indicated by seahorse analysis. MAPK inhibition restores glycolytic activities (b), whereas inhibition of both, PI3K and MAPK, reduced oxidative phosphorylation to the levels observed in wiNPCs (c) ( $n=4$ group/condition with four technical replicates). (d) Inhibition of glycolysis by 2-DG compromises self-renewal properties in transformed iNPCs ( $n=4$ group with four technical replicates). (e) Chemical induced glycolysis inhibition (2-DG) and ROS production (Rot/AA) compromises transformed iNPC viability in MTS assays ( $n=4$ group/condition with four technical replicates). Data are represented as mean \pm s.d. $P$ values were calculated by Student's $t$-test. ${ }^{\star} P<0.05$. 
the identified compounds alongside metabolic modulators in a more physiological setting. To this end, we relied on the use of ex vivo organotypic brain slices, $5,38,39$. Injection of primary GTICs into $300 \mu \mathrm{m}$ brain slices resulted in the formation of well-defined tumour masses in less than 8 days (Fig. 4d). Treatment of brain slices indicated a specific effect against primary GTIC tumours with 2 -DG, nelarabine and letrozole, demonstrating the highest anti-tumour potential (Fig. 4d,e).

\section{Discussion}

Cancer arises as a consequence of transforming 'driver' mutations able to initiate tumour formation. Upon tumour initiation, positive selection and clonal progression further leads to the accumulation of 'passenger' mutations conferring additional growth advantages. Identification of driver mutations might therefore allow not only for the establishment of targeted therapies but also, most importantly, for the elucidation and targeting of the early events underlying cancer formation. Although enormous progress has been made, current bioinformatics approaches tend to focus on diver mutations found in high frequencies while underestimating the role that low frequency mutations might play during carcinogenesis ${ }^{40-45}$.

Since their discovery, iPSCs have been widely used for the modelling of genetic disorders and multiple reports have highlighted the suitability of reprogramming as a platform for drug discovery studies ${ }^{18,36}$. In an analogous manner, different strategies have been used for the modelling of human cancer ${ }^{2,3,10-15}$ including the generation of induced pluripotent cancer cells by reprogramming of cancer cells to a pluripotent state ${ }^{46,47}$ and the transformation of differentiated fibroblast by oncogenes and tumour suppressor genes to a cancer stem cell-like phenotype ${ }^{37}$. More recently, conversion of differentiated cancer cells to a multipotent cancer stem cell phenotype upon overexpression of factors defining GTIC identity has been reported ${ }^{3}$. Reprogramming of differentiated glioma cells to glioma stem cells opens the unprecedented opportunity for investigating the dynamic dedifferentiation processes leading to the appearance of GTICs (ref. 3). However, the need for primary tumour material (or glioma cell lines) in where transforming mutations are already present could prevent studies on the role that specific mutations might play during gliomagenesis. Directly addressing the lack of model for studying driver mutations, the Tabar laboratory first reported on the use of NPCs differentiated from human ESCs for studying the role that histone 3.3 mutations play in the formation of pediatric gliomas ${ }^{2}$. Along a similar line, our own results demonstrate the suitability of hiPSCs technologies for the establishment of GTIC-like models recapitulating features observed in adult human gliomas.

In summary, here we report on the application of iPSC technologies for the establishment of tractable human GTIC-like models in vitro and in vivo. Similar to conventional disease modelling strategies based on the use of hiPSCs, the establishment of hiPSC cancer models might facilitate the future development of novel therapeutics ${ }^{46-48}$.

\section{Methods}

Reagents and antibodies. The following antibodies were used at the specified concentrations for flow cytometry analysis: mouse anti-human TRA-1-60-FITC 1:10 (560380, BD), mouse anti-human TRA-1-81-APC 1:10 (560793, BD), mouse anti-human CD184 (CXCR4)-APC 1:10 (555976, BD), mouse anti-human CD15-PE and CD15-FITC (561715 and 555401, BD), mouse anti-human CD24-PE 1:10 (560991, BD), mouse anti-human SSEA4-AlexaFluor647 1:10 (560219, BD), mouse anti-human CD44-PE 1:10 (550989, BD), mouse anti-human CD133/2 (293C3)-PE 1:10 (130-090-853, Miltenyi), mouse APC isotype control 1:10 (555751, BD), mouse FITC isotype control 1:10 (555748, BD), mouse PE isotype control 1:10 (555749, BD).

The following antibodies were used at the specified concentrations for immunostainings: sex-determining region Y-box 2 (SOX2; AB5603, 1:500 Chemicon); Oct-3/4 (sc-5279, 1:500; Santa Cruz Biotechnology); NANOG (EB06860, 1:500; Everest Biotech); NANOG (21624, 1:400, abcam); anti-phospho-Akt Ser473 (\#9271, 1:50; Cell Signaling); anti-phospho-ERK1/2 Thr202/Tyr204 (\#9101, 1:50; Cell Signaling); neuron-specific class III $\beta$-tubulin (\#MRB-435p and MMS-435p,

TUJ-1; 1:500; Covance); microtubule-associated protein 2 (\#AB5622, MAP2; 1:500; Millipore); PAX6 (PRB-278P; 1:1000; Covance), glial fibrillary acidic protein (\#Z0334, GFAP; 1:500; Dako); $\alpha 1$-fetoprotein (A0008, AFP; 1:400; Dako); calponin (M3556, 1:500; Dako); Ki67 (ab16667; 1:500; abcam); NESTIN (MAB5326, 1:500; Millipore); human nuclear antigen (\#MAB1281, HUNU; 1:250, Millipore); O4 (MAB345, 1:200; Milipore), PECAM-1 (\#sc-1506, CD31; 1:50; Santa Cruz Biotechnology); 4,6-diamidino-2-phenylindole (DAPI) $\left(5 \mathrm{mg} \mathrm{ml}^{-1}\right)$ 1:2,000 (D1306 Invitrogen, Carlsbad, CA); and Alexa Fluor 488 goat anti-mouse (1:1,000, Invitrogen), Alexa Fluor 488 donkey anti-goat (1:1,000, Invitrogen), Alexa Fluor 488 donkey anti-rabbit (1:1,000, Invitrogen) Alexa Fluor 568 donkey anti-mouse (1:1,000, Invitrogen), Alexa Fluor 568 donkey anti-rabbit (1:1000, Invitrogen) and Alexa Fluor 647 chicken anti-rabbit (1:1,000, Invitrogen).

The following chemicals were used at the specified concentrations: TGF $\beta$ inhibitor SB431542 $10 \mu \mathrm{M}$ (Reagents Direct; \#21-A94); PI3K inhibitor LY294002 $10 \mu \mathrm{M}$ (Sigma-Aldrich, St Louise, MO; \#L9908); PD032591 MAPK inhibitor $1 \mu \mathrm{M}$ (Axon medichem; \#Axon140); CHIR99021 $3 \mu \mathrm{M}$ (Axon Medichem; \#Axon1386); MMP-9 Inhibitor I $10 \mathrm{nM}$ (Santa Cruz Biotechnology; \#sc-311-437); FCCP $0.5 \mu \mathrm{M}$ Rotenone + Antimycin A $1 \mu \mathrm{M}$ and $0.1 \mu \mathrm{M}$ (Rot/AA) and 2-Deoxy-D-glucose $5 \mathrm{mM}$ (2-DG) (Sigma). DMSO was used as a solvent and as a vehicle control in the respective experiments.

Cell culture. Neonatal human fibroblasts (HFF-1; ATCC), 293T and the human glioma lines U-87 MG and LN229 (ATCC) were cultured in DMEM containing $10 \% \mathrm{FBS}, 2 \mathrm{mM}$ GlutaMAX (Invitrogen), $50 \mathrm{U} \mathrm{ml}^{-1}$ penicillin and $50 \mathrm{mg} \mathrm{ml}^{-1}$ streptomycin (Invitrogen). Human foreskin fibroblasts (HFF) were grown in collagen I coated plates (BD biosciences). Human iPSCs were cultured in chemically defined hES/hiPSCs growth media, mTeSR on growth factor reduced matrigel (BD biosciences) coated plates. Briefly, 70-80\% confluent iPSCs cells were treated with dispase (Invitrogen) for $7 \mathrm{~min}$ at $37^{\circ} \mathrm{C}$ and the colonies were dispersed to small clusters and lifted carefully using a 5 -ml glass pipette at a ratio of $\sim 1: 4$ GTICs, previously reported and characterized ${ }^{5}$, were obtained from BioRep S.r.l. (NS27Z + B AB0789) and maintained in culture as described elsewhere ${ }^{5}$. All cell lines were maintained in an incubator $\left(37^{\circ} \mathrm{C}, 5 \% \mathrm{CO} 2\right)$ with media changes every day (iPSCs) or every second day (fibroblasts, 293T cells, GTICs).

Plasmids. pMX-OCT4, pMX-SOX2, pMX-KLF4 and pMX-cMYC, were obtained from Addgene (plasmids 17217, 17218, 17219 and 17220, respectively). The shRNA $p 53$ construct was previously described and validated ${ }^{49}$. Constructs for the expression of mutant oncogenes were obtained from Addgene (RasV12 Neo (w108-1) \#22259; EGFR D770-N771 insNPG \#11016; EGFR (del3)

Figure 4 | Anti-cancer compound screening identifies chemicals specifically targeting transformed iNPCs and primary GTICs. (a) Annexin $V$ staining, as measured by flow cytometry, validates the role of lead compounds at the indicated concentrations and demonstrates a specific effect against primary GTICs and transformed iNPCs for three of the compounds analysed (Nelarabine, Letrozole and Capecitabine). In contrast, Cabazitaxel demonstrated group-dependent effects and targeting of wTiNPCs. Data are presented as a ratio to the values obtained in the respective DMSO control groups. Negative values indicate increase viability in where basal Annexin $V$ staining was higher in DMSO samples as compared with those treated with specific compounds; ( $n=3$ group/condition with three technical replicates). (b,c) Treatment of transformed iNPCs and primary GTICs with the identified compounds and metabolic modulators compromises glioma stem cell properties including migration ( $\mathbf{b}, n=3$ group/condition with three technical replicates) and selfrenewal potential in single-cell assays ( $\mathbf{c} n=3$ group/condition with 24 technical replicates). (d) Tumour size measured on brain organotypic cultures for each indicated condition. Data are independently plotted for each organotypic brain slice analysed ( $n=>3$ condition with technical triplicates). (e) Representative pictures of organotypic brain slices injected with primary GTICs and treated with each of the indicated compound. Brain slices were immunostained with the indicated markers as follows: DAPI (blue), GFP (green) and Ki67 (red). Data are represented as mean \pm s.d. $P$ values were calculated by Student's $t$-test or Mann-Whitney test when appropriate. ${ }^{\star} P<0.05$. Scale bars, $100 \mu \mathrm{m}(\mathbf{e})$. 
\#11015; src Y527F \#13660). Coding sequence for the mutant oncogenes were removed by restriction enzymes and subcloned into a lentiviral backbone with the In-Fusion system according to manufacturer's instructions

(Takara/Clontech).
Retroviral and lentiviral production. Moloney-based retroviral vectors (pMXs) were co-transfected with packaging plasmids (pCMV-gag-pol-PA and pCMV-

VSVg) in 293T cells using Lipofectamine 2000 (Invitrogen) according to

manufacturer's instructions. Retroviral supernatants were collected $48 \mathrm{~h}$ after

a
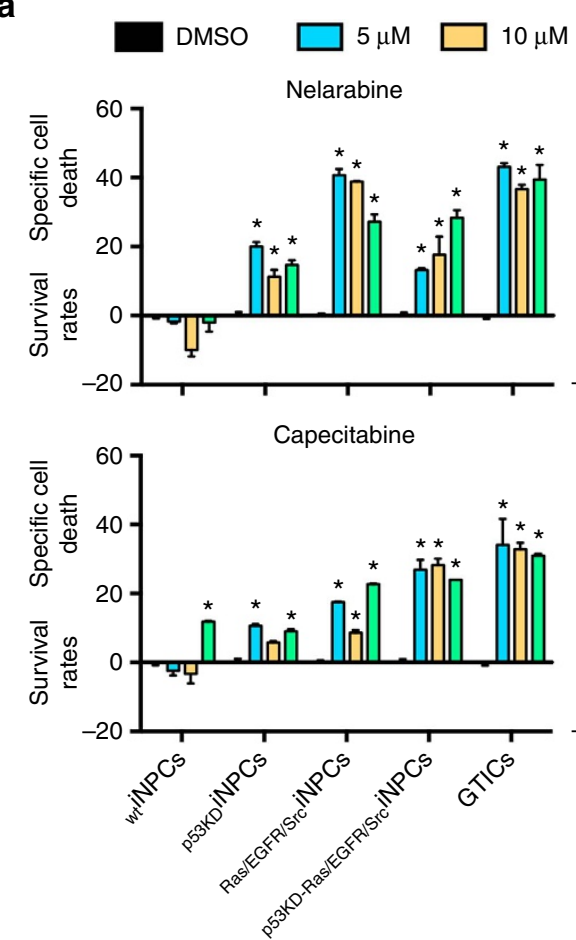

$\square 20 \mu \mathrm{M}$
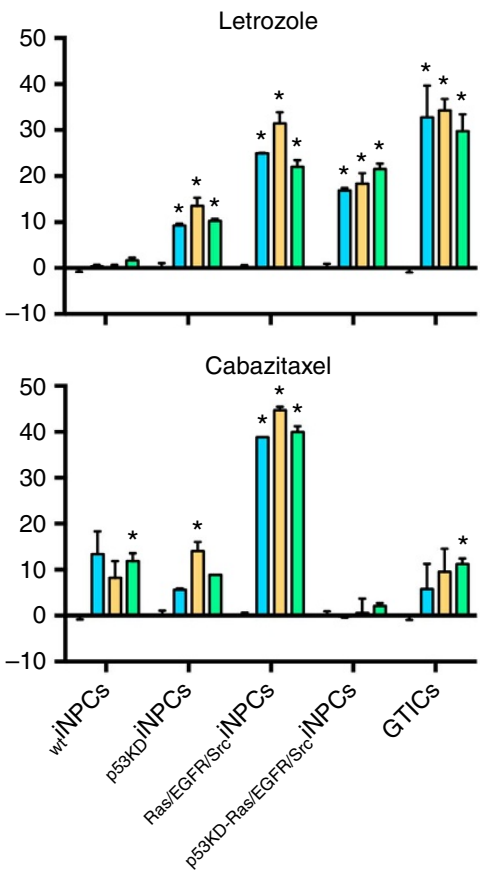

b

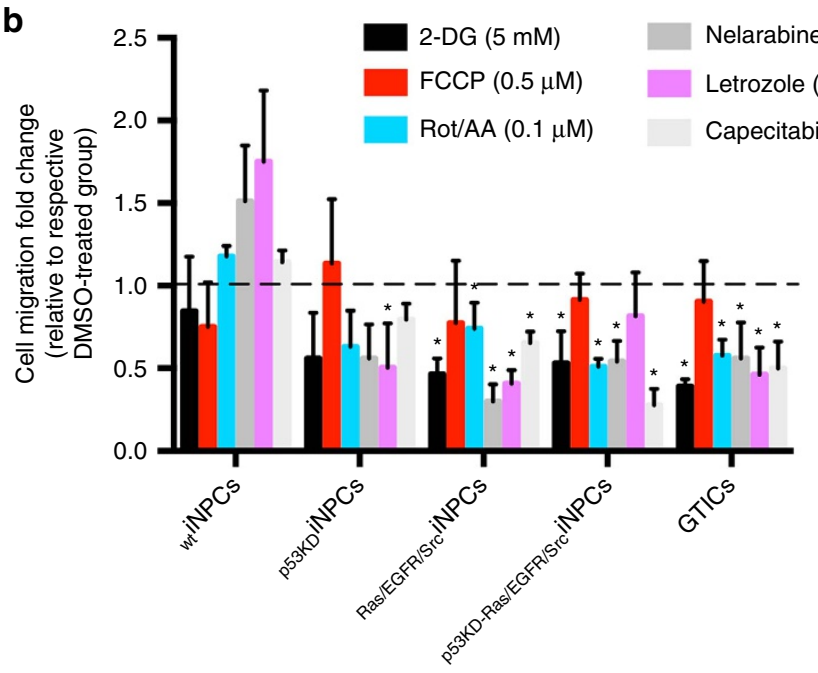

d

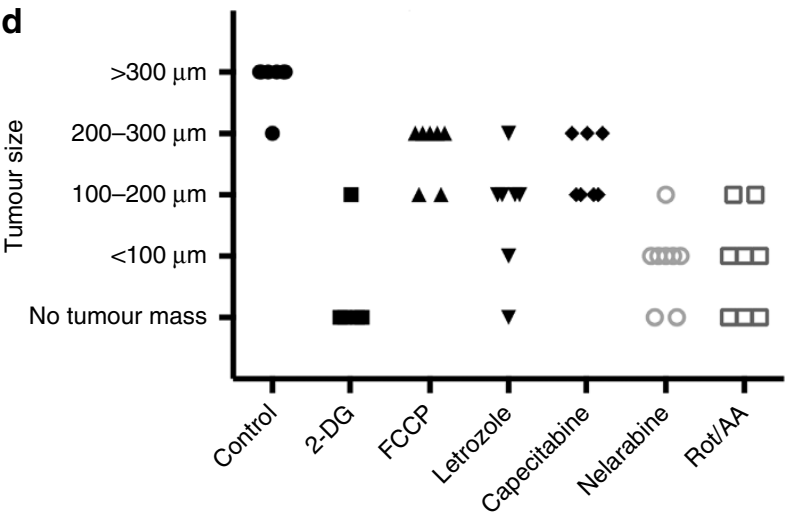

e

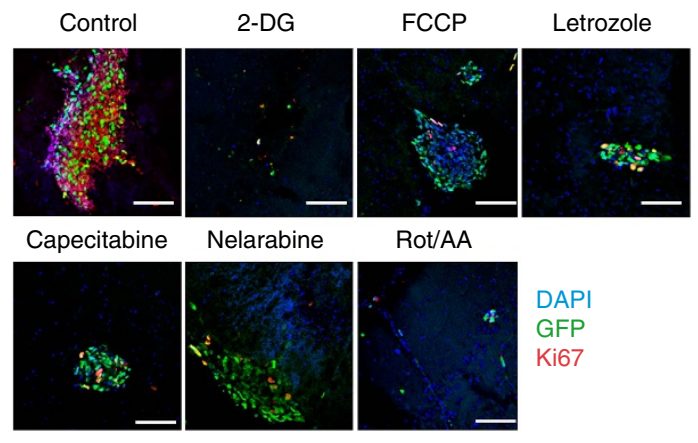

C

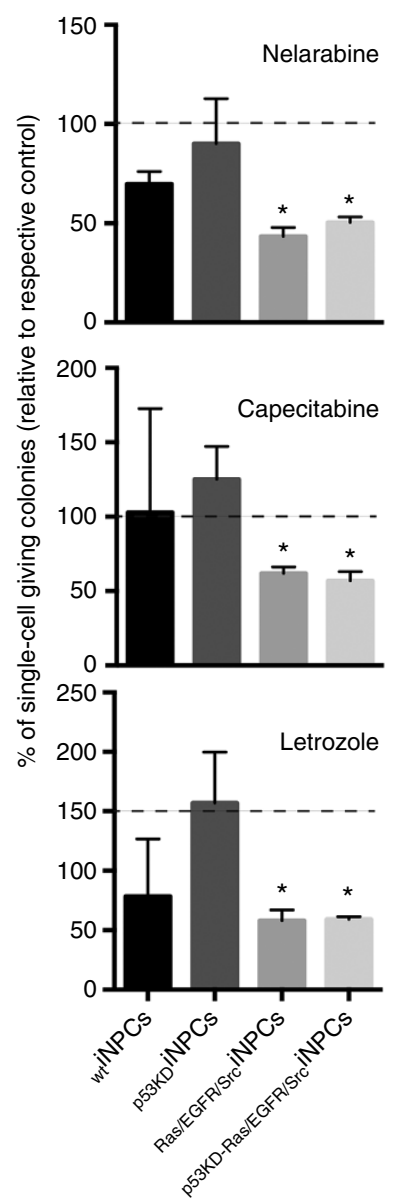


transfection, passed through a $0.45 \mu \mathrm{M}$ filter to remove cellular debris and stored at $4{ }^{\circ} \mathrm{C}$ for up to 7 days. Lentiviral vectors were co-transfected with packaging plasmids (pMDL, Rev and VSVg) in 293T cells using Lipofectamine 2000 (Invitrogen) according to manufacturer's instructions. Lentiviral supernatants were collected $48 \mathrm{~h}$ after transfection, concentrated by ultracentrifugation at 19400 r.p.m. for $2 \mathrm{~h}$, resuspended in PBS and stored at $-80^{\circ} \mathrm{C}$.

Induced pluripotent stem cell generation and subculture. For the generation of human iPS cells, primary HFF were infected with an equal ratio of retroviruses (Oct4, SOX2, KLF4 and c-MYC) by spinfection of the cells at 1850 r.p.m. for $1 \mathrm{~h}$ at room temperature in the presence of polybrene $\left(4 \mu \mathrm{g} \mathrm{ml}^{-1}\right)$. After two serial infections, cells were passaged onto fresh mouse embryonic fibroblasts and switched to hES medium containing DMEM/F12 (Invitrogen) supplemented with $20 \%$ Knockout Serum Replacement (Invitrogen), $1 \mathrm{mM}$ L-glutamine, $0.1 \mathrm{mM}$ non-essential amino acids, $55 \mathrm{M}$-mercaptoethanol and $10 \mathrm{ng} \mathrm{ml}^{-1} \mathrm{bFGF}$ (preprotech). For the derivation of hiPS cells lines, iPS-like colonies were manually picked and maintained on fresh mouse embryonic fibroblast feeder layers for five passages before being transferred onto Matrigel $/ \mathrm{mTesR} 1$ conditions $\left(n=2 \mathrm{wT}_{\mathrm{T}} \mathrm{PSC}\right.$ clones). iPSCs with a knockdown of p53 were generated as described ${ }^{49}$. Briefly, HFF were infected with lentiviral particles expressing a shRNA against p53 before iPSC reprogramming ( $n=3$ p53KD iPSC clones).

Embryoid body differentiation. Embryoid bodies (EBs) were produced from adherent colonies that were enzymatically detached using dispase $\left(0.5 \mathrm{mg} \mathrm{ml}^{-1}\right)$ for $15-45 \mathrm{~min}$, collected and then maintained in suspension using ultra low attachment plates (corning) in the presence of EB medium containing DMEM/F12 supplemented with $15 \%$ fetal bovine serum, $2 \mathrm{mM}$ L-glutamine, $0.1 \mathrm{mM}$ nonessential amino acids, and $1 \%$ penicillin/streptomycin. After 3-4 days, EBs were transferred to $0.1 \%$ gelatin-coated polystyrene chamber slides and cultured in differentiation medium (DMEM supplemented with $20 \%$ fetal bovine serum, $2 \mathrm{mM}$ L-glutamine, $0.1 \mathrm{mM} 2$-mercaptoethanol, $0.1 \mathrm{mM}$ non-essential amino acids and $1 \%$ penicillin/streptomycin) for 2-3 weeks to allow spontaneous endoderm formation. The medium was changed every other day. For mesoderm differentiation, EBs were maintained on gelatin-coated plate in differentiation medium supplemented with $100 \mu \mathrm{M}$ ascorbic acid (Sigma). For ectoderm differentiation, EBs were cultured on Matrigel-coated plates in 1\% N2 and $0.5 \%$ B27 (Invitrogen) medium supplemented with $1 \mu \mathrm{M}$ retinoic acid for 2-3 weeks.

Derivation of iNPCs from iPSCs. Neural progenitor cell (NPC) induction was based on a previous report with slight modifications ${ }^{50}$. The day before the start of induction, hiPSCs (wTiPSCs and ${ }_{\mathrm{p} 53 \mathrm{KD}} \mathrm{iPSCs}$ ) were passaged onto matrigel-coated plates at about $20 \%$ confluence and maintained in mTesR overnight. At day 0 , culture medium was then switched to Neural Induction Medium 1 (NIM-1: 50\% Advanced DMEM/F12 (Invitrogen), 50\% Neurobasal (Invitrogen), $1 \times \mathrm{N} 2$ (Invitrogen), $1 \times$ B27 (Invitrogen), $2 \mathrm{mM}$ GlutaMAX (Invitrogen) and $10 \mathrm{ng} \mathrm{ml}^{-1}$ hLIF (Millipore), $4 \mu \mathrm{M}$ CHIR99021 (Cellagentech, premade in $10 \mathrm{mM}$ DMSO solution), $3 \mu \mathrm{M}$ SB431542 (Cellagentech, premade in $10 \mathrm{mM}$ DMSO solution), $2 \mu \mathrm{M}$ Dorsomorphin (Sigma) and $0.1 \mu \mathrm{M}$ Compound E (EMD Chemicals Inc.). Cells were treated with NIM-1 for 2 days, and then switched to Neural Induction Medium 2 (NIM-2: 50\% Advanced DMEM/F12, 50\% Neurobasal, $1 \times$ N2, $1 \times$ B27, 2 mM GlutaMAX, $10 \mathrm{ng} \mathrm{ml}^{-1}$ hLIF, $4 \mu \mathrm{M}$ CHIR99021, $3 \mu \mathrm{M}$ SB431542 and $0.1 \mu \mathrm{M}$ Compound E) for another 5 days. The cultures were then split onto Matrigel-coated plates with Accumax (Innovative Cell Technologies) and cultured in Neural Stem cell Maintenance Medium (NSMM) containing 50\% Advanced DMEM/F12, 50\% Neurobasal, $1 \times \mathrm{N} 2,1 \times$ B27, $2 \mathrm{mM}$ GlutaMAX, $10 \mathrm{ng} \mathrm{ml}^{-1}$ hLIF, $3 \mu \mathrm{M}$ CHIR99021 and $2 \mu \mathrm{M}$ SB431542.

iNPC culture and multilineage neural differentiation. iNPCs were maintained on Matrigel in NSMM and passaged once $80-100 \%$ confluent using Accumax. Medium was changed every day. For the initial six passages, iNPCs were treated with $10 \mu \mathrm{M}$ Y-27632 (Biomol Inc.) during splitting. For neuronal differentiation, $\sim 1 \times 10^{5}$ human iNPCs cells were plated onto poly-ornithine (Sigma) and laminin coated 35-mm dishes in NSMM medium. After 2 days, medium was switched to Neurobasal media (Invitrogen) supplemented with N2 (Invitrogen), B27 (Invitrogen) and FGF2 (10 $\mathrm{ng} \mathrm{ml}^{-1}$; Peprotech). Four days later, FGF2 was withdrawn from the medium, and after another 4 days, medium was switched to Neurobasal media supplemented with B27 and brain derived neurotrophic factor (BDNF, $20 \mathrm{ng} \mathrm{ml}^{-1}$, R\&D Systems). Differentiated cells were maintained up to 4 weeks after FGF2 withdrawal. For astrocyte generation, cells were treated with $5 \%$ serum for 2-3 weeks.

iNPC transformation. Three days before lentiviral transduction, iPSC-derived NPCs ( ${ }_{\mathrm{WT}} \mathrm{iNPCs}$ and ${ }_{\mathrm{P} 53 \mathrm{KD}} \mathrm{iNPCs}$, passages 6-8) were passaged. On the day of transduction, each of the four lentiviral preparations (RasV12, EGFR D770-N771; EGFR (del3) and src Y527F) was thawed on a bed of ice, mixed at a 1:1:1:1 ratio (MOI of 0.75 per virus) in NSMM medium in the presence of polybrene $\left(4 \mu \mathrm{g} \mathrm{ml}^{-1}\right)$. NPCs culture medium was replaced with the lentiviruses containing medium and cells were incubated overnight at $37^{\circ} \mathrm{C}$. The next day, the medium was replaced with fresh NSMM. Transduced iNPCs (Ras/EGFR/SrciNPCs, P53KD-Ras/ EGFR/SrciNPCs were passaged for at least two more passages for stabilization before performing analyses.

Flow cytometry analysis. For flow cytometry analysis, cells were harvested using TrypLE (Invitrogen), washed once with PBS and further incubated with the corresponding antibodies in the presence of FACS blocking buffer $(1 \times \mathrm{PBS} / 10 \% \mathrm{FCS})$ for $1 \mathrm{~h}$ on ice in the absence of light. After incubation, cells were washed thrice with $1 \mathrm{ml}$ of FACS blocking buffer and resuspended in a total volume of $200 \mu \mathrm{l}$ before analysis using an LSRII instrument (Becton-Dickinson, Fullertone, CA). A minimum of 10,000 cells in the living population was analysed. Percentages are presented after subtracting isotype background and referring to the total living population analysed. Results are representative of at least two independent experiments with a minimum of two technical replicates per experiment $(n \geq 4)$ and per condition.

For cell death assays, iNPCs and primary GTICs were treated with 5, 10 and $20 \mu \mathrm{M}$ for the indicated chemotherapeutic compounds in duplicates in six-wellplates. Human glioma lines (U-87 MG and LN229) were treated with $1 \mathrm{mM}$ for the indicated compounds. $24 \mathrm{~h}$ after treatment, cells were dissociated by Accutase (Innovative Cell Technologies, cat. no. AT-104) and Annexin V/PI staining conducted following the manufacturer's recommendations (88-8007-72 Annexin V APC Ebiosciences). PI staining served to exclude dead cells and only Annexin V cells present in the PI negative living cell population (indicative of early apoptosis) were considered for analysis. All stainings were done in biological triplicate with technical triplicates ( $n=9$ total) per line and per condition.

Cell sorting. Transformed iNPCs were sorted for CD15, CD133 and CXCR4 cells using anti-CD15 (130-046-601; Miltenyi biotec), anti-CD133 (130-097-049; Miltenyi biotec) and anti-CXCR4 (130-100-070; Miltenyi biotec) conjugated magnetic beads according to the manufacturer's instructions with slight modifications. Briefly, up to $10^{9}$ cells were incubated with constant mixing at $4{ }^{\circ} \mathrm{C}$ with $100 \mu \mathrm{l}$ of the corresponding magnetic beads in the presence of $100 \mu \mathrm{l}$ of Fc-blocking solution in a total volume of $500 \mu \mathrm{l}$ FACS blocking buffer. After $1 \mathrm{~h}$, cells were sorted by two consecutive rounds of column separation in order to increase purity by applying MACS separation magnets. Shortly, cells were passed through the first MS separation column allowing binding of labelled cells. Non-labelled cells were washed thoroughly with $3 \mathrm{ml}$ fluorescence-activated cell sorting blocking buffer before elution of the labelled fraction. Eluted labelled cells were then subjected to a second purification step as described above. Both positive and negative fractions were collected for further analyses.

Boyden chamber migration assays. Cells were treated with $10 \mu \mathrm{g} \mathrm{ml}^{-1}$ Mitomycin C (Sigma) for $1 \mathrm{~h}$ before the assay. Cells were rinsed once with $1 \times$ PBS (Invitrogen), and treated with TrypLE (Invitrogen) for $5 \mathrm{~min}$ at $37^{\circ} \mathrm{C}$. TrypLE was neutralized with DMEM/F12 (Invitrogen) $+10 \%$ FBS (HyClone). Cells were centrifuged at 1000 r.p.m. for $5 \mathrm{~min}$, and resuspended in their respective basal media. A total of 20,000 cells was seeded onto the upper chamber of each transwell (BD). $1 \mathrm{ml}$ culture media was applied to the lower chamber of each transwell. Migration assay was performed for $24 \mathrm{~h}$ in an incubator set at $37^{\circ} \mathrm{C}$ in the presence or absence of MMP-9 Inhibitor I (10 nM), the metabolic modulators 2-DG (5 mM), FCCP $(0.5 \mu \mathrm{M})$, Rot/AA $(0.1 \mu \mathrm{M})$ and the chemotherapeutic compounds Nelarabine $(10 \mu \mathrm{M})$, letrozole $(10 \mu \mathrm{M})$ and capecitabine $(10 \mu \mathrm{M})$. The cells attached onto the upper side of the transwell filter were removed by a cotton stick. The migrated cells attached onto the bottom side of the transwell filter were fixed with 4\% PFA (Sigma), and visualized with $0.5 \%$ crystal violet staining (Sigma). Five different areas of each chamber were randomly selected for photography with $\times 5$ objective (Olympus). The average cell number/area was calculated for each chamber. Biological Triplicates with technical triplicates $(n=9)$ were performed for each cell line.

In vitro self-renewal and single-cell assays. Transformation assays were conducted by modifying standard soft-agar protocols for the culturing of iNPCs similar to what was previously described ${ }^{51}$. Briefly, $2 \times 10^{4}$ cells were individualized, embedded into $2 \%$ matrigel and seeded in $60-\mathrm{mm}$ dishes. Biological triplicates with technical triplicates $(n=6)$ were performed for each tested cell line and condition.

For single-cell self-renewal assays the experimental setup was modified as follows: undifferentiated (sorted or unsorted) iNPCs were dissociated and plated into matrigel-coated 96 -well plates as single cells as described ${ }^{52}$. Each condition was done in 24 technical replicates with a minimum of three biological duplicates $(n \geq 62)$. One day after plating, all wells were treated with the respective compounds as indicated. After 21 days, the colony number was evaluated by microscopy analysis using an upright microscope (Olympus). For single-cell selfrenewal assays in the presence of PI3K (LY294002; $5 \mu \mathrm{M}$ ) and MAPK (PD98059; $5 \mu \mathrm{M})$ inhibitors, individual cells were plated as described above and the media supplemented with the respective chemicals or the solvent control (DMSO) at the indicated concentration and colonies counted after 21 days. Biological triplicates with technical triplicates $(n=9)$ were performed for each tested cell line and condition. 
Single-cell kill assays were conducted by plating individual iNPCs onto 96-well plates as single cells before differentiation. Differentiation into neuronal lineages was performed as described above in the presence of the indicated chemicals or the respective DMSO controls. Cell survival was estimated with trypan blue after differentiation over 28 days. Each condition was done in 24 replicates with a minimum of two independent times $(n \geq 48)$.

Immunoblotting. Cells were solubilized in lysis buffer $(50 \mathrm{mM}$ Tris- $\mathrm{HCl}(\mathrm{pH} 7.4)$, $10 \%$ Glycerol, $1 \%$ (v/v) Triton X-100, $100 \mathrm{mM} \mathrm{NaCl}, 0.5 \mathrm{mM} \mathrm{MgCl}_{2}, 1 \mathrm{mM}$ $\mathrm{Na}_{3} \mathrm{VO}_{4}, 10 \mu \mathrm{g} \mathrm{ml}^{-1}$ aprotinin, $1 \mathrm{mM}$ PMSF, $1 \mathrm{mM}$ pepstatin and leupeptin), and the resulting lysates were prepared by centrifugation at $16,000 \mathrm{~g}$ for $15 \mathrm{~min}$ at $4{ }^{\circ} \mathrm{C}$. The protein concentration of cell lysates was tested using the protein assay reagent (Bio-Rad) before immunoblotting. Protein samples were separated on SDS-polyacrylamide gel electrophoresis, and transferred onto PVDF membrane filters (PALL). The following primary antibodies were used according to the manufacturer's instructions: anti-phospho-Akt Ser473 (\#9271, Cell Signaling), anti-Akt (\#9272, Cell Signaling), anti-phospho-ERK1/2 Thr202/Tyr204 (\#9101, Cell Signaling), anti-ERK1/2 (\#9102, Cell Signaling), anti-NANOG (\#ab21624, Abcam), anti- $\alpha$-Tubulin (\#T5168, Sigma) and anti- $\beta$-Actin (\#sc-47778, Santa Cruz). Uncropped images of the most important immunoblots are provided in Supplementary Fig. 11.

RNA isolation and real-time PCR analysis. Total cellular RNA was isolated using Trizol Reagent (Invitrogen) according to the manufacturer's recommendations. An amount of $1 \mu \mathrm{g}$ of DNAsel (Invitrogen) -treated total RNA was used for cDNA synthesis using the iScript cDNA synthesis kit for PCR with reverse transcription (BioRad). Real-time PCR was performed using the SYBR Green Supermix (BioRad). The levels of expression of respective genes were normalized to corresponding GAPDH values and are shown as fold change relative to the value of the control sample. All samples were done in biological triplicate with a minimum of two technical replicates $(n \geq 5)$. The list of the primers used for real-time PCR experiments are listed in the Supplementary Table 5.

\section{Metabolic flux analysis. Analysis of oxygen consumption rate (OCR) and} extracellular acidification rate (ECAR) was performed using a Seahorse extracellular flux XF96 analyser (Seahorse Bioscience, Billerica, MA) in accordance with manufacturer's instructions. Briefly, cells were seeded at a density of $1.5 \times 10^{4}$ cells per well in Matrigel-treated extracellular flux 96-well culture microplates in $150 \mu \mathrm{l}$ of corresponding cell culture media and incubated overnight at $37^{\circ} \mathrm{C}$. The next day, before analysis, culture media was replaced with $150 \mu$ l of unbuffered assay media and cells were incubated for $1 \mathrm{~h}$ at $37^{\circ} \mathrm{C}$ for $\mathrm{pH}$ and temperature stabilization. Analysis of OCR and ECAR was performed simultaneously both at basal conditions and after injections of the inhibitors in the XF Cell Mito Stress Test Kit $\left(1 \mu \mathrm{g} \mathrm{ml}^{-1}\right.$ Oligomycin, $0.5 \mu \mathrm{M}$ FCCP, $1 \mu \mathrm{M}$ Antimycin A $+1 \mu \mathrm{M}$ Rotenone (Sigma)). To evaluate the effect of the respective PI3K and MAPK inhibitors LY294002 and PD0325901 on cellular metabolism, cells were incubated overnight $(12 \mathrm{~h})$ in the presence of $10 \mu \mathrm{M}$ LY294002 or $1 \mu \mathrm{M}$ PD0325901. The next day, before analysis, culture media was replaced and analysis of OCR and ECAR was performed as described above. Each condition was done in four technical replicates with a minimum of four biological replicates $(n \geq 16)$.

MTS assays. Analysis of cellular viability in the presence of respiratory inhibitors was performed using MTS assay (Promega, G3580) according to the manufacturer's instructions. Briefly, cells were seeded at a density of $2 \times 10^{4}$ cells per well in Matrigel-treated 96-well culture microplates in $100 \mu \mathrm{l}$ of culture media and incubated for $4 \mathrm{~h}$ at $37^{\circ} \mathrm{C}$. Next, the mitochondrial respiratory inhibitors FCCP or $0.5 \mu \mathrm{M}$ Antimycin A $+0.5 \mu \mathrm{M}$ Rotenone (Sigma) were added at the indicated concentrations. After $24 \mathrm{~h}$ of compound/DMSO treatment, $20 \mu \mathrm{l}$ of MTS reagent was applied to each well of 96 -well plate. Absorbance at $490 \mathrm{~nm}$ was recorded after $2 \mathrm{~h}$ incubation. Sextuplets were prepared for each condition.

ROS measurements. Mitochondrial-associated ROS levels and total intracellular ROS levels were measured by staining cells with MitoSOX and $\mathrm{H}_{2}$ DCFDA. For mitochondrial superoxide, cells were washed with PBS solution, trypsinized and resuspended in PBS in the presence of $5 \mu \mathrm{M}$ MitoSOX (Invitrogen) for $15 \mathrm{~min}$ at $37^{\circ} \mathrm{C}$. Subsequently, cells were washed twice with PBS solution containing $10 \%$ FBS and analysed by FACS using an LSRII instrument (Becton-Dickinson). For total intracellular ROS, cells were washed with PBS solution and incubated in HBSS solution in the presence of $5 \mu \mathrm{M} \mathrm{H}_{2} \mathrm{DCFDA}$ (Invitrogen) for $30 \mathrm{~min}$ at $37^{\circ} \mathrm{C}$. Afterwards, the HBSS solution was replaced with regular media and cells were incubated for an additional $1 \mathrm{~h}$ at $37^{\circ} \mathrm{C}$. Lastly, cells were washed with PBS solution, trypsinized and resuspended in PBS solution containing 10\% FBS for FACS analysis using an LSRII instrument (Becton-Dickinson). As a positive control, cells were incubated in the presence of the mitochondrial inhibitor Antimycin A (Sigma) at a concentration of $0.1 \mu \mathrm{M}$ for $30 \mathrm{~min}$ at $37^{\circ} \mathrm{C}$ to induce ROS production. Each condition was done in two technical replicates with three biological replicates $(n=6)$.
Mitochondrial staining. For mitochondrial staining, cells were seeded on Matrigel-treated cover slips at the appropriate densities. The next day, cells were incubated for $30 \mathrm{~min}$ at $37^{\circ} \mathrm{C}$ in regular media in the presence of $200 \mathrm{nM}$ MitoTracker Red (Invitrogen) for staining of mitochondria and $1 \mu \mathrm{g} \mathrm{ml}^{-1}$ DAPI (Invitrogen) to stain the cell nuclei. Thereafter, cells were fixed in $4 \%$ paraformaldehyde and visualized by a confocal microscopy (Zeiss, Oberkochen, Germany) with excitation at $578 \mathrm{~nm}$ and emission at $598 \mathrm{~nm}$ for MitoTracker Red, and excitation at $359 \mathrm{~nm}$ and emission at $461 \mathrm{~nm}$ for DAPI detection. Each condition was done in two technical replicates with three biological replicates $(n=6)$.

\section{Gas chromatography/mass spectrometry/stable isotope labelling. For} GC-MS profiling analysis of intracellular metabolites, cells were seeded and cultured in 6-well dishes (to about 1 million cells / $0.5 \mathrm{mg}$ cell protein per well). Cells were washed quickly three times with cold PBS, and $0.45 \mathrm{ml}$ cold methanol $(50 \% \mathrm{v} / \mathrm{v}$ in water with $20 \mu \mathrm{M}$-norvaline as internal standard) was added to each well. Culture plates were transferred to dry ice for $30 \mathrm{~min}$. After thawing on ice, the methanol extract was transferred to a microcentrifuge tube. Chloroform $(0.225 \mathrm{ml})$ was then added, the tube was vortexed and centrifuged at $10,000 \mathrm{~g}$ for $5 \mathrm{~min}$ at $4{ }^{\circ} \mathrm{C}$ The upper layer was transferred to another microcentrifuge tube, dried in a centrifugal evaporator and derivatized with $30 \mu \mathrm{l} O$-isobutylhydroxylamine hydrochloride $\left(20 \mathrm{mg} \mathrm{ml}^{-1}\right.$ in pyridine, TCI) for $20 \mathrm{~min}$ at $80^{\circ} \mathrm{C}$, followed by $30 \mu \mathrm{l}$ $\mathrm{N}$-tert-butyldimethylsilyl- $\mathrm{N}$-methyltrifluoroacetamide (Sigma) for $60 \mathrm{~min}$ at $80^{\circ} \mathrm{C}$ After cooling overnight at $4^{\circ} \mathrm{C}$, the mixture was then transferred to SHIMADZU GCMS-QP2010 Plus for analysis.

GC-MS protocols and analysis were similar to those described before ${ }^{53}$, except a modified temperature gradient was used for GC: Initial temperature was $130^{\circ} \mathrm{C}$ held for $4 \mathrm{~min}$, rising at $6^{\circ} \mathrm{C} \mathrm{min}-1$ to $243^{\circ} \mathrm{C}$, rising at $60^{\circ} \mathrm{C} \mathrm{min}-1$ to $280^{\circ} \mathrm{C}$, held for $2 \mathrm{~min}$. Data were processed as before with quantification against varied amounts of standard mixtures run in parallel performed using MetaQuant. Quantities were corrected for recovery using the L-norvaline internal standard.

For labelling experiments, cells were seeded as described above. Then cells were changed to NSMM, with $50 \%{ }^{13} \mathrm{C}$-glucose for labelling. The glucose was isotopically labelled at all six carbon positions ([U- $\left.{ }^{13} \mathrm{C}_{6}\right]$ glucose). After $24 \mathrm{~h}$ of incubation, labelled cells were rinsed with cold PBS. Cells were then processed for GC/MS sample extraction and derivatization, and analysed as described before ${ }^{53,54}$.

For normalization, protein quantification was determined. Cells from separate and replicate wells for GC/MS were rinsed once with ice-cold PBS and lysed in ice-cold lysis buffer ( $25 \mathrm{mM}$ Tris (pH 8.0), $100 \mathrm{mM} \mathrm{NaCl}, 1 \%$ TritonX-100 and $10 \%$ Glycerol) and one tablet of EDTA-free protease inhibitors (Roche; per $25 \mathrm{ml}$ ). The soluble fractions of cell lysates were isolated by centrifugation at 13,000 r.p.m. for 15 min. Protein concentration was determined by the Bradford method with Bio-Rad DC Protein Assay Kit (Hercules, CA, USA).

Animals. Murine experiments presented hereafter were conducted with approval of The Salk Institute Institutional Animal Care and Use Committee (IACUC), under the protocol\#08-025. NOD.Cg-PrkdcscidIl2rgtm1Wjl /SzJ mice (or NODScid IL2r rnull abbreviated as NSG; age, 7 weeks; males and females; weight, $20 \mathrm{~g}$ ) were purchased from the Jackson Laboratory, housed in air-flow racks on a restricted access area and maintained on a 12-h light/dark cycle at a constant temperature $\left(22 \pm 1{ }^{\circ} \mathrm{C}\right)$. For all experiments involving the use of animals, no inclusion/exclusion criteria were applied in the present study.

Teratoma assay. Severe combined immune NSG male mice $(n=2$ animal/iPS clone; 5 clones total), $\sim 8$ weeks old, were injected with iPSCs ( 1 million for each injection site, approximately) subcutaneously in the testicular parenchyma. Additionally, undifferentiated iPSCs were stereotactically injected into the brain of immunocompromised NSG mice ( $n=2$ animal/iPS clone; five clones total). Mice were sacrificed 8 weeks after the injections or when a tumour was detected by palpation or when animals displayed signs of distress, whichever came first. Teratoma formation was assessed by immunofluorescence techniques. Randomization was unnecessary and no blinding was done.

In vivo tumour formation. For cell preparation, cells were rinsed once with $1 \mathrm{XPBS}$ (Invitrogen), and treated with TrypLE (Invitrogen) for $5 \mathrm{~min}$ at $37^{\circ} \mathrm{C}$. TrypLE was neutralized with DMEM/F12 (Invitrogen) $+10 \%$ FBS. Cells were centrifuged at 1000 r.p.m. for $5 \mathrm{~min}$, and resuspended in PBS $\left(2 \times 10^{5} \mu \mathrm{l}^{-1}\right.$ or $500 \mathrm{\mu l}^{-1}$ for $5 \times 10^{5}$ and 500 transplanted cells, respectively). Cells were kept on a bed of ice before transplantation. For cell transplantation, anaesthetized NSG mice were inserted in a stereotactic frame, the skull surface was exposed and a hole was drilled at the appropriate site to allow single/unilateral (right hemisphere) injection of the cell preparations. Antero-posterior (AP), lateral (L) and vertical (V) coordinates (in $\mathrm{mm}$ ) for cell transplantations were taken relative to the bregma: $\mathrm{AP}+0.5$, $\mathrm{L}+2, \mathrm{~V}-1.5$. For cell injections, a $10 \mu \mathrm{l}$ Hamilton syringe was used and the flow rate set at $\sim 0.5 \mu \mathrm{l} \mathrm{min}^{-1}\left(2.5 \mu \mathrm{l}\right.$ or $0.5 \mu \mathrm{l}$ animal for $5 \times 10^{5}$ and 500 transplanted cells, respectively). For each condition, five animals were injected. Randomization was unnecessary and no blinding was done.

For secondary transplantation experiments, primary recipient animals were sacrificed by carbon dioxide (CO2) overdose and their brains immediately removed. The tumour was then extracted under stereomicroscope with surgical 
tools, in sterile conditions. The tumour mass was cut into small pieces and placed in TrypLE solution for $15 \mathrm{~min}$ at $37^{\circ} \mathrm{C}$. The tumour was fully dissociated by mechanical dissociation to obtain a single-cell solution, and passed through a $0.45 \mu \mathrm{m}$ filter. Then, the cells were prepared and injected into immunocompromised mice as described above. Of note, tumour extraction, cell preparation and cell injection were performed the same day. For each condition, five animals were injected. Randomization was unnecessary and no blinding was done.

Animal perfusion/tissue sectioning. Animals were daily monitored and sacrificed/perfused when showing cues of sickness (that is, dehydrated, lethargic, scruffy and/or hunched). For perfusion, mice were sacrificed by carbon dioxide (CO2) overdose and then transcardially injected with $20 \mathrm{ml}$ ice-cold saline $\left(0.9^{0} \%\right.$ $\mathrm{NaCl}$ ), followed by $50 \mathrm{ml}$ ice-cold $4 \%$ paraformaldehyde (PFA, pH 7.4; $4^{\circ} \mathrm{C}$ ). Brains were then extracted and post-fixed in PFA overnight at $4{ }^{\circ} \mathrm{C}$. Thirty-six to forty hours before sectioning, brains were cryoprotected in a 30\% sucrose solution. Frozen coronal sections $(35 \mu \mathrm{m}$ thickness) were cut with a cryostat (Leica) and were either collected onto positively charged slides or kept at $-20^{\circ} \mathrm{C}$ into an ethylenglycol-based cryoprotectant solution until being processed for immunostainings. Before performing cryosections, whole brain pictures were taken with a camera.

Haematoxylin/eosin staining. Tissue sections on positively charged slides were processed for haematoxylin-eosin staining by dipping the slides in a series of baths as follow: distilled water ( $30 \mathrm{~s})$, haematoxylin ( $5 \mathrm{~min})$; distilled water (1 $\mathrm{min}) ; 0.5 \%$ eosin Y (2 min); $95 \%$ Ethanol $(30 \mathrm{~s} \times 2)$; $100 \%$ ethanol $(30 \mathrm{~s} \times 2)$; and xylene $(30 s \times 2)$. Depex mounting medium and glass cover slips were used to mount the slides. Slides were analysed by using an upright microscope (Olympus).

Immunofluorescence analyses. Briefly, cells were washed thrice with PBS and fixed using $4 \%$ PFA in $1 \times$ PBS for $12 \mathrm{~min}$ and then washed three times in PBS. For tissue analysis, fixed floating sections maintained in a cryoprotectant solution were washed in PBS before being immunostained. Fixed cells/tissues were blocked and permeabilized for $1 \mathrm{~h}$ at RT with $3 \% \mathrm{BSA} / 5 \%$ appropriate serum/ $1 \times$ PBS in the presence of $0.1 \%$ Triton X-100 and $0.3 \mathrm{M}$ glycine. Subsequently, cells/tissue sections were incubated with the indicated primary antibody either for $1 \mathrm{~h}$ at room temperature or overnight at $4{ }^{\circ} \mathrm{C}$. Cells/tissue sections were then washed thrice with $1 \times$ PBS and incubated for $1 \mathrm{~h}$ at RT with the respective secondary antibodies and $20 \mathrm{~min}$ with DAPI. Cells/tissue sections were washed thrice with $1 \times$ PBS before analysis. Cells/tissue sections were analysed by confocal microscopy. Confocal image acquisition was performed using a Zeiss LSM 780 or LSM 710 laser scanning microscope (Carl Zeiss).

Gene expression analysis. For gene expression analyses, RNA was purified using the RNeasy Plus mini kit (74134, Qiagen). Purity and integrity of the RNA samples were assessed by spectrophotometry and nanoelectrophoresis using the NanoDrop ND-1000 spectrophotometer (NanoDrop Technologies, Wilmington, DE) and the Nano lab-on-a-chip assay for total eukaryotic RNA using Bioanalyser 2100 (Agilent Technologies, Palo Alto, CA), respectively. Only samples with high purity and high integrity were subsequently used in microarray experiments.

Microarray expression profiles were obtained using the Affymetrix GeneChip Human Gene 1.0 ST Array (Affymetrix, Santa Clara, CA). Amplification, labelling and hybridizations were performed according to protocols from Ambion (Ambion/ Applied Biosystems, Foster City, CA, USA) and Affymetrix (Affymetrix Inc.). Briefly, $200 \mathrm{ng}$ of total RNA were amplified using the Ambion WT Expression Kit (Ambion/Applied Biosystems), labelled using the WT Terminal Labeling Kit (Affymetrix) and then hybridized to Human Gene 1.0 ST Array (Affymetrix) for $16 \mathrm{~h}$ at $45^{\circ} \mathrm{C}$ and 60 r.p.m. in a GeneChip Hybridization Oven 640. Following hybridization, the array was washed and stained in the Affymetrix GeneChip Fluidics Station 450 . The stained array was scanned using an Affymetrix GeneChip Scanner 30007 G, generating CEL files for each array. Normalized and log transformed gene expression values were filtered using a variance filter $(\sigma / \sigma \max )$ of 0.2. Each variable was standardized by subtraction of its mean value and division

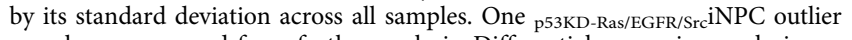
sample was removed from further analysis. Differential expression analysis was carried out using ANOVA in Qlucore Omics Explorer 2.3. Probes with a $q$ value $<0.01$ were considered differentially expressed. The differentially expressed probes were then clustered using hierarchical clustering and only the probes $(n=463)$ that displayed high correlation between the GTICs and ${ }_{\text {p53KD-Ras/EGFR/SrciNPC samples }}$ were displayed in the heatmap. Pathways analysis was performed using IPA software (Ingenuity Systems, build v. 308606 M, content v. 18488943).

Chip-Seq and methylation analysis. ChiP-Seq data was mapped and loaded into Seqmonk tools (Babraham Institute) and duplicate reads were removed. Peaks were defined as a contiguous set of reads that were enriched by at least 10 -fold. A 50 base pair gap was allowed to prevent large peaks from being broken into multiple small peaks. The number of reads in each peak were counted, corrected for total read count and log transformed. An intensity difference test was used to identify differential SOX2-binding sites. An intensity difference test is a pairwise statistical test using the general distribution of the data and tests whether a particular point is likely to be an outlier when compared with the overall distribution of the data. The test takes each point and tests a subset of points from the pair of samples being examined and then constructs a distribution of the differences between the two data sets. The distribution is then compared with a normal distribution allowing for a $P$ value to be calculated. A FDR of 0.05 was used as a cutoff. Genes within $100 \mathrm{~kb}$ of the differentially bound peak were considered to be associated with SOX2-binding events.

For methylation analyses, DNA was purified by using DNeasy kit (69504, Qiagen), quantified (Qubit dsDNA BR Assay, Life Technologies) and bisulfiteconverted (EZDNA Methylation Kit, Zymo Research) according to the manufacturer's protocol. Bisulfite-converted DNA was then labelled and hybridized to the Infinium Human Methylation $450 \mathrm{~K}$ beadchip (Illumina) and scanned on a HiScan (Illumina). Samples were normalized and filtered using the statistical programming language R (http://www.r-project.org/) (v.3.0.1) and the R package minfi (v.1.6.0). Briefly, samples were first control-normalized using Illumina's internal bead controls and probes with a detection $P$ value $>0.01$ in at least one sample were discarded. The samples were then SWAN normalized using the minfi package and $\beta$ values (Methylated allele intensity/ Unmethylated allele intensity + methylated allele intensity) were exported. Probes with less than a max $\beta-\min \beta$ of 0.5 were removed.

Compound screening. 101 anti-cancer compounds were provided by the National Cancer Institute-chemotherapeutic agents repository. A full list is provided in Supplementary Table 7. For MTS assays, 96-well plates were coated with matrigel (BD, cat. no. 354230) in a $37^{\circ} \mathrm{C}$ cell culture incubator for $2 \mathrm{~h}$. iNPCs and GTICs were enzymatically dissociated by Accutase (Innovative Cell Technologies, cat. no. AT-104). Live cell number was counted using a hemocytometer in the presence of Trypan Blue (Gibco, cat. no. 15250). For each iNPC line, 30,000 live cells were resuspended in $50 \mu \mathrm{l}$ NSMM and seeded into one well of 96-well plate. For GTICs, 15,000 live cells were resuspended in $50 \mu \mathrm{l} \mathrm{NSMM}$ and seeded into one well of 96 -well plate. The cells were allowed to grow in a $37^{\circ} \mathrm{C}$ cell culture incubator for $24 \mathrm{~h}$ before compound/DMSO treatment. Compound/DMSO were diluted in NSMM (final concentration of $10 \mu \mathrm{M}$ ) and added into each well to make a 100- $\mu \mathrm{l}$ final volume. After $24 \mathrm{~h}$ compound/DMSO treatment, $20 \mu \mathrm{l}$ MTS (Promega, G3580) was applied to each well of 96-well plate. Absorbance at $490 \mathrm{~nm}$ was recorded after $2 \mathrm{~h}$ incubation. Quadruplets were prepared for one compound/line. For Flow Cytometry analysis, cells were treated in the presence of 5, 10 and $20 \mu \mathrm{M}$ in duplicates in six-well-plates. $24 \mathrm{~h}$ after treatment cells were dissociated by Accutase (Innovative Cell Technologies, cat. no. AT-104) and Annexin V/PI staining conducted following the manufacturer's recommendations (88-8007-72 Annexin V APC Ebiosciences). PI staining served to exclude dead cells and only Annexin V cells present in the PI negative living cell population (indicative of early apoptosis) were considered for analysis. All stainings were done in biological and technical triplicates per line and per condition.

Brain organ cultures. Brain organotypic slices were obtained from adult male NSG mice as previously described ${ }^{55,56}$. Briefly, $3-8,300 \mu \mathrm{m}$ slices per murine brain were obtained with a Leica VT1200 microtome and cultured over $0.4 \mu \mathrm{m}$ air-liquid interface membranes (Millipore) for $24 \mathrm{~h}$. 200,000 cells were then injected and tumour formation allowed for the next $72 \mathrm{~h}$. Brain slices were then incubated in the presence of the indicated chemicals or the respective DMSO control for $72 \mathrm{~h}$ before immunofluorescence analysis. Each condition was done in two technical replicates with a minimum of three biological replicates $(n \geq 6)$.

Statistical evaluation. Statistical analyses were performed by using SPSS/PC + statistics 11.0 software (SPSS Inc., Chicago, IL). All data presented a normal distribution. Statistical significance was evaluated using standard unpaired Student's $t$ test (two-tailed, 95\% confidence intervals, $P<0.05$ ) with Welch's correction when appropriate. For multiple comparison analysis, one-way ANOVA with Dunnett's or Bonferroni correction post-test was applied when appropriate $(P<0.05)$. Comparisons of groups with small sample size $(n<6)$ were performed as follows: (i) Mann-Whitney test (two-sided, 95\% confidence level; $P<0.05$ ) was used; (ii) Kruskal-Wallis test with Dunn's post-test $(P<0.05)$ has been applied for multiple comparisons. All data are presented as mean \pm s.d. and represent a minimum of two independent experiments with at least two technical replicates.

\section{References}

1. Chen, J., McKay, R. M. \& Parada, L. F. Malignant glioma: lessons from genomics, mouse models, and stem cells. Cell 149, 36-47 (2012).

2. Funato, K., Major, T., Lewis, P. W., Allis, C. D. \& Tabar, V. Use of human embryonic stem cells to model pediatric gliomas with $\mathrm{H} 3.3 \mathrm{~K} 27 \mathrm{M}$ histone mutation. Science 346, 1529-1533 (2014).

3. Suva, M. L. et al. Reconstructing and reprogramming the tumor-propagating potential of glioblastoma stem-like cells. Cell 157, 580-594 (2014).

4. Medema, J. P. Cancer stem cells: the challenges ahead. Nat. Cell Biol. 15, 338-344 (2013). 
5. Pollard, S. M. et al. Glioma stem cell lines expanded in adherent culture have tumor-specific phenotypes and are suitable for chemical and genetic screens. Cell Stem Cell 4, 568-580 (2009).

6. Flavahan, W. A. et al. Brain tumor initiating cells adapt to restricted nutrition through preferential glucose uptake. Nat. Neurosci. 16, 1373-1382 (2013).

7. Magee, J. A., Piskounova, E. \& Morrison, S. J. Cancer stem cells: impact, heterogeneity, and uncertainty. Cancer Cell 21, 283-296 (2012).

8. Visvader, J. E. \& Lindeman, G. J. Cancer stem cells in solid tumours: accumulating evidence and unresolved questions. Nat. Rev. Cancer 8, 755-768 (2008).

9. Visvader, J. E. \& Lindeman, G. J. Cancer stem cells: current status and evolving complexities. Cell Stem Cell 10, 717-728 (2012).

10. Koso, H. et al. Transposon mutagenesis identifies genes that transform neural stem cells into glioma-initiating cells. Proc. Natl Acad. Sci. USA 109, E2998-E3007 (2012).

11. Sonoda, Y. et al. Formation of intracranial tumors by genetically modified human astrocytes defines four pathways critical in the development of human anaplastic astrocytoma. Cancer. Res. 61, 4956-4960 (2001).

12. Sonoda, Y. et al. Akt pathway activation converts anaplastic astrocytoma to glioblastoma multiforme in a human astrocyte model of glioma. Cancer. Res. 61, 6674-6678 (2001)

13. Marumoto, T. et al. Development of a novel mouse glioma model using lentiviral vectors. Nat. Med. 15, 110-116 (2009).

14. Friedmann-Morvinski, D. et al. Dedifferentiation of neurons and astrocytes by oncogenes can induce gliomas in mice. Science 338, 1080-1084 (2012).

15. Mao, X. G. et al. LIN28A facilitates the transformation of human neural stem cells and promotes glioblastoma tumorigenesis through a pro-invasive genetic program. Oncotarget 4, 1050-1064 (2013).

16. Lang, M. F. et al. Genome-wide profiling identified a set of miRNAs that are differentially expressed in glioblastoma stem cells and normal neural stem cells. PLoS ONE 7, e36248 (2012).

17. Takahashi, K. \& Yamanaka, S. Induction of pluripotent stem cells from mouse embryonic and adult fibroblast cultures by defined factors. Cell 126, 663-676 (2006).

18. Robinton, D. A. \& Daley, G. Q. The promise of induced pluripotent stem cells in research and therapy. Nature 481, 295-305 (2012).

19. Miller, J. \& Studer, L. Aging in iPS cells. Aging (Albany NY) 6, 246-247 (2014).

20. Miller, J. D. et al. Human iPSC-based modeling of late-onset disease via progerin-induced aging. Cell Stem Cell 13, 691-705 (2013).

21. Brennan, C. W. et al. The somatic genomic landscape of glioblastoma. Cell 155, 462-477 (2013).

22. Ahluwalia, M. S., de Groot, J., Liu, W. M. \& Gladson, C. L. Targeting SRC in glioblastoma tumors and brain metastases: rationale and preclinical studies. Cancer. Lett. 298, 139-149 (2010).

23. Han, X. et al. The role of Src family kinases in growth and migration of glioma stem cells. Int. J. Oncol. 45, 302-310 (2014).

24. Huveldt, D. et al. Targeting Src family kinases inhibits bevacizumab-induced glioma cell invasion. PLoS ONE 8, e56505 (2013).

25. Kleber, S. et al. Yes and PI3K bind CD95 to signal invasion of glioblastoma. Cancer Cell 13, 235-248 (2008).

26. Singh, S. K. et al. Identification of human brain tumour initiating cells. Nature 432, 396-401 (2004).

27. Wang, J. et al. CD133 negative glioma cells form tumors in nude rats and give rise to CD133 positive cells. Int. J. Cancer 122, 761-768 (2008).

28. Nishide, K., Nakatani, Y., Kiyonari, H. \& Kondo, T. Glioblastoma formation from cell population depleted of Prominin1-expressing cells. PLoS ONE 4, e6869 (2009).

29. Zhu, L. et al. Prominin 1 marks intestinal stem cells that are susceptible to neoplastic transformation. Nature 457, 603-607 (2009).

30. Gilbert, C. A. \& Ross, A. H. Cancer stem cells: cell culture, markers, and targets for new therapies. J. Cell. Biochem. 108, 1031-1038 (2009).

31. Vander Heiden, M. G., Cantley, L. C. \& Thompson, C. B. Understanding the Warburg effect: the metabolic requirements of cell proliferation. Science 324, 1029-1033 (2009)

32. Mustafa, D. A., Swagemakers, S. M., Buise, L., van der Spek, P. J. \& Kros, J. M. Metabolic alterations due to IDH1 mutation in glioma: opening for therapeutic opportunities? Acta Neuropathol. Commun. 2, 6 (2014).

33. Ricci-Vitiani, L. et al. Tumour vascularization via endothelial differentiation of glioblastoma stem-like cells. Nature 468, 824-828 (2010).

34. Zhou, C. et al. Proteomic analysis of tumor necrosis factor-alpha resistant human breast cancer cells reveals a MEK5/Erk5-mediated epithelial-mesenchymal transition phenotype. Breast Cancer Res. 10, R105 (2008).

35. Visvader, J. E. \& Lindeman, G. J. Stem cells and cancer-the promise and puzzles. Mol. Oncol. 4, 369-372 (2010).

36. Sachlos, E. et al. Identification of drugs including a dopamine receptor antagonist that selectively target cancer stem cells. Cell 149, 1284-1297 (2012).
37. Scaffidi, P. \& Misteli, T. In vitro generation of human cells with cancer stem cell properties. Nat. Cell. Biol. 13, 1051-1061 (2011).

38. Arai, K., Nishiyama, N., Matsuki, N. \& Ikegaya, Y. Neuroprotective effects of lipoxygenase inhibitors against ischemic injury in rat hippocampal slice cultures. Brain. Res. 904, 167-172 (2001).

39. Cho, S., Wood, A. \& Bowlby, M. R. Brain slices as models for neurodegenerative disease and screening platforms to identify novel therapeutics. Curr. Neuropharmacol. 5, 19-33 (2007).

40. Krogan, N. J., Lippman, S., Agard, D. A., Ashworth, A. \& Ideker, T. The cancer cell map initiative: defining the hallmark networks of cancer. Mol. Cell 58, 690-698 (2015).

41. Hartmaier, R. J., Priedigkeit, N. \& Lee, A. V. Who's driving anyway? Herculean efforts to identify the drivers of breast cancer. Breast. Cancer. Res. 14, 323 (2012).

42. Raphael, B. J., Dobson, J. R., Oesper, L. \& Vandin, F. Identifying driver mutations in sequenced cancer genomes: computational approaches to enable precision medicine. Genome Med. 6, 5 (2014).

43. Ding, L., Wendl, M. C., McMichael, J. F. \& Raphael, B. J. Expanding the computational toolbox for mining cancer genomes. Nat. Rev. Genet. 15, 556-570 (2014).

44. Gonzalez-Perez, A. et al. Computational approaches to identify functional genetic variants in cancer genomes. Nat. Methods 10, 723-729 (2013).

45. Tamborero, D. et al. Comprehensive identification of mutational cancer driver genes across 12 tumor types. Sci. Rep. 3, 2650 (2013).

46. Curry, E. L., Moad, M., Robson, C. N. \& Heer, R. Using induced pluripotent stem cells as a tool for modelling carcinogenesis. World J. Stem Cells 7, 461-469 (2015).

47. Nishi, M. et al. Induced cancer stem-like cells as a model for biological screening and discovery of agents targeting phenotypic traits of cancer stem cell. Oncotarget 5, 8665-8680 (2014).

48. Tiscornia, G., Vivas, E. L. \& Izpisua Belmonte, J. C. Diseases in a dish: modeling human genetic disorders using induced pluripotent cells. Nat. Med. 17, 1570-1576 (2011).

49. Kawamura, T. et al. Linking the p53 tumour suppressor pathway to somatic cell reprogramming. Nature 460, 1140-1144 (2009).

50. Li, W. et al. Rapid induction and long-term self-renewal of primitive neural precursors from human embryonic stem cells by small molecule inhibitors. Proc. Natl Acad. Sci. USA 108, 8299-8304 (2011).

51. Spike, B. T. et al. A mammary stem cell population identified and characterized in late embryogenesis reveals similarities to human breast cancer. Cell Stem Cell 10, 183-197 (2012).

52. Kurian, L. et al. Conversion of human fibroblasts to angioblast-like progenitor cells. Nat. Methods 10, 77-83 (2013).

53. Scott, D. A. et al. Comparative metabolic flux profiling of melanoma cell lines: beyond the Warburg effect. J. Biol. Chem. 286, 42626-42634 (2011).

54. Ma, L. et al. Control of nutrient stress-induced metabolic reprogramming by PKCzeta in tumorigenesis. Cell 152, 599-611 (2013).

55. Stoppini, L., Buchs, P. A. \& Muller, D. A simple method for organotypic cultures of nervous tissue. J. Neurosci. Methods 37, 173-182 (1991).

56. Filipovic, R., Kumar, S. S., Bahr, B. A. \& Loturco, J. Slice culture method for studying migration of neuronal progenitor cells derived from human embryonic stem cells (hESC). Curr. Protoc. Stem Cell Biol. 29, 1H 7 1-1H 714 (2014).

\section{Acknowledgements}

We thank M. Schwarz for administrative support. Y.X. were partially supported by a postdoctoral training grant from the California Institute for Regenerative Medicine (CIRM). E.N. was partially supported by a F.M. Kirby Foundation postdoctoral fellowship. I.S.-M. was partially supported by a Nomis Foundation postdoctoral fellowship. O.A. is supported by the NIHR Oxford Biomedical Research Centre. We thank the staff at the Institute for Genomic Medicine Genomics Facility at UCSD for running the Infinium HumanMethylation450 BeadChips. We acknowledge the San Diego Supercomputing Center (through an allocation from the eXtreme Science and Engineering Discovery Environment [XSEDE]) for providing HPC resources that have contributed to the research results reported within this paper. We thank David Scott and the Cancer Metabolism Shared Resource in the Sanford-Burnham Medical Research Institute Cancer Center (supported by the NCI under award number 5P30CA030199) for assistance with GC-MS and ${ }^{13} \mathrm{C}$-labelling experiments. L.M. and I.D. were partially supported by UCAM. R.M. and L.C.L. were supported by the UCSD Department of Reproductive Medicine. G.M.W., I.M.V. and J.C.I.B. are supported by the Cancer Center Core Grant (P30 CA014195-38) and the Leona M. and Harry B. Helmsley Charitable Trust Grant \#2012-PG-MED002. I.M.V. is supported in part by grants from the NIH (HL053670) and the H.N. and Frances C. Berger Foundation. Work in the laboratory of J.C.I.B. was supported by grants from NIH (5U01HL107442), CIRM, UCAM and the G. Harold and Leila Y. Mathers Charitable Foundation. 


\section{Author contributions}

I.S.-M. and E.N. conceived the initial study. Y.X., I.S.-M., E.N. and J.C.I.B. designed all experiments except when indicated. I.S.-M., E.N. and J.C.I.B. wrote the manuscript. Y.X., I.S.-M. and E.N. performed and analysed all experiments. E.N. performed all animals experiments and in vitro experiments. I.S.-M. performed all Flow Cytometry stainings. I.S.M. and Y.X. conducted anti-cancer compound screening. Y.X. performed western blotting and migrations experiments. M.N.K., I.D., C.R.E. and E.N. performed teratoma experiments. O.A. analysed brain tumours. M.N.K. and A.Z. performed organotypic brain experiments. T.H., E.V.-F. and Y.H. produced all constructs and performed self-renewal assays. A.O., P.R., D.L. and L.M. performed metabolic studies. A.A. performed immunofluorescence studies. R.M. and L.C.L. generated and analysed all bioinformatic data. M.W. provided reagents. G.M.W., D.O. and I.M.V. contributed to the overall design of the project and revised the manuscript.

\section{Additional information}

Accession codes: Differential ChiP-Seq, Methylation and Gene Expression data have been deposited in GEO under the accession code GSE67286.
Supplementary Information accompanies this paper at http://www.nature.com/ naturecommunications

Competing financial interests: The authors declare no competing financial interests.

Reprints and permission information is available online at http://npg.nature.com/ reprintsandpermissions/

How to cite this article: Sancho-Martinez, I. et al. Establishment of human iPSC-based models for the study and targeting of glioma initiating cells. Nat. Commun. 7:10743 doi: 10.1038/ncomms10743 (2016).

\section{(c) (i)}

This work is licensed under a Creative Commons Attribution 4.0 International License. The images or other third party material in this article are included in the article's Creative Commons license, unless indicated otherwise in the credit line; if the material is not included under the Creative Commons license, users will need to obtain permission from the license holder to reproduce the material. To view a copy of this license, visit http://creativecommons.org/licenses/by/4.0/ 